\title{
Health, Education and Endogenous Growth
}

\author{
by \\ A.H. van Zon ${ }^{*}+$ \\ and \\ J. Muysken + \\ Maastricht, April 1997, First Draft
}

Keywords: Health, Education, Endogenous Growth Abstract

The purpose of the paper is to show that, from a growth perspective, government resources can be spent in two different ways. Resources can be allocated to uses which support growth, and to uses which generate growth. We take the provision of health services as an example of the first type of use, and the provision of educational services as an example of the second. This enables us to integrate both types of uses of scarce resources in an endogenous growth framework and to derive the optimum mix of the provision of health and educational services both from the perspective of health as a complement to growth and health as a substitute for growth. The model illustrates that there is a trade-off between growth as such and the provision of health-services. It also shows that a slow down in growth could be expected to occur when the preference for health is positively influenced by a growing income per head or in the case of an ageing population. Finally, we show that the model can account for a 'growth take off' in countries which are too poor to save, and that this take off can be induced by 'just the right' amount of income transfer to those countries : too little aid doesn't seem to help at all, while too much aid unnecessarily burdens the long term solvability of the receiving country if aid is provided in the form of loans.

* MERIT, Maastricht Economic Research Institute on Innovation and Technology, PO Box 616, 6200 MD Maastricht, the Netherlands

+ Faculty of Economics and Business Administration, Maastricht University, PO Box 616, 6200 MD Maastricht, the Netherlands

E-mail: Adriaan.vanZon@MERIT.UNIMAAS.NL, Tel. +33 (0)43 3883890, Fax +33

(0)433216518 


\section{Introduction}

Baumol (1967) stresses the importance of technological differences between the separate sectors of an economy for its growth performance at the aggregate level. More in particular, Baumol divides economic activities into those which do not provide any serious scope for productivity increases (like personal services) and those which do (industrial activities in particular). He concludes that as a result of these productivity asymmetries, the value share of 'low productivity' activities will gain in importance over time, i.e. a growing part of national income will be spent (and earned) through low productivity activities, as long as there is a demand for these activities. This phenomenon has become known as 'Baumols disease'.

A typical example of such a low productivity personal service may be the provision of health services. Since health problems are almost always experienced as acute by the sufferer, the demand for health services will not vanish as long as people are subject to (exogenous) variations in their health status. Moreover, the low productivity character of the health sector is enhanced because the provision of health services is subject to decreasing returns to scale. ${ }^{1}$ Hence both conditions for the occurrence Baumols disease are fulfilled. An indication of the practical relevance of Baumols observations is the fact that the already significant value share of the health sector in the West is increasing with national income. ${ }^{2}$

From a growth perspective, one may wonder whether the health sector would provide a drag on growth performance by directly competing for scarce resources with the 'knowledge generation sectors' which are central to endogenous growth as brought forward in Lucas (1988) and Romer (1990), for instance. On the other hand one may argue that a good health is a prerequisite for economic growth and hence should be stimulated by all means. Thus one could envisage the existence of a growth trade-off, since an expansion of the health sector would promote growth through increased health, while a contraction of the health sector could also free the resources necessary to promote growth by means of an expansion of the knowledge producing sector.

In order to analyse this trade-off and its consequences for economic growth, we extend the endogenous growth framework of Lucas and Romer mentioned earlier. In this framework the process of knowledge accumulation and the provision of health services take up scarce human capital resources. As indicated above, the framework reflects the productivity asymmetries present in Baumol (1967), where the health sector is taken as an example of a sector which permits ' ... only sporadic increases in productivity'

1 The decreasing returns assumption with respect to the provision of health services is present in models of the demand for health in, for instance, Muurinen (1982), Forster (1989), Ehrlich and Chuma (1990) and Johansson and Lofgren (1995).

2 See Lapre and Rutten (1993), for instance. On page 32, Lapre and Rutten provide a figure which shows that the value share of expenditures on health in national income rises with national income per head, and that it is highest for the US with an average share of about 10 percent in the period 1977-1982, and somewhat lower for other countries, among which the Netherlands with an average of about 8 percent in the same period. On page 33, the relevance of Baumols observations for the health sector is further underlined when they state that the increase in the value share of the health sector can in part be attributed to the relatively low rate of labour productivity growth in the health sector, which lags seriously behind the growth of labour productivity in the agricultural and industrial sectors, but also in the commercial services sector. 
because'... there is no substitute for the personal attention of a physician ...' ${ }^{3}$ as opposed to the knowledge producing sector, which, in the terminology of Baumol, is engaged in '... technologically progressive activities in which innovations, capital accumulation, and economies of large scale make for a cumulative rise in output per man hour'. (Baumol 1967, pp. 416,423,415, respectively). The obvious question now is what the optimum allocation of the macro-economic budget is over its four different uses : consumption, physical capital accumulation, knowledge accumulation and the provision of health services, in the face of (differences in the valuation of) the intertemporal effects of these activities.

In order to answer this question, we build two endogenous growth models based on the Lucas (1988) model, in which health influences intertemporal decision making in three different ways. First, it serves as the conditio sine qua non to the provision of human capital services. Second, the provision of health services directly competes with the provision of labour services allocated to the direct generation of output, but also with the provision of labour services allocated to knowledge generation. In our model we assume that the provision of labour services depends both on the average level of health of the work force and the stock of knowledge per worker. The idea is that a deterioration of health either reduces the quality of the labour services rendered, or, alternatively, it reduces the number of effective working days. Seen in this way, health and knowledge are complements, in that a low health status will lead to a low supply of human capital services, ceteris paribus, but, from the perspective of the generation of effective human capital services, the provision of health services is also a substitute for the generation of human capital per se. We show that in this set-up there is an optimum mix of the provision of health and educational services. The third way in which health influences intertemporal decision making follows from the observation that health can generate positive utility of its own. When we include this notion in our first model, it follows that attaching a positive weight to having a good health lowers the optimum rate of growth of the economy.

Actually, we solve the second model by employing a graphical analysis which offers the possibility to illustrate what policy induced changes in the productivity characteristics of the health and educational sector would mean for growth, health and welfare in general. As an illustration of the model, we run two simulations. The first simulation shows how this model can explain Baumols disease by linking the preference for health to per capita income, and by allowing part of the population to become unproductive due to 'ageing'. The second simulation shows the impact of an income transfer to a poor country with a low average level of health. Here we assume the preference for health to depend positively on income per head and the rate of discount to depend negatively on income per head. Thus we arrive at the conclusion that there may exist an optimum level of transfers.

The remainder of the paper is organised as follows. Section 2 provides a short summary of the main features of the Lucas (1988) model, and the 'health generation' module which we want to combine with that model. Section 3 describes two different extensions of the Lucas model in which health is introduced next to the accumulation of knowledge. Section 4 provides an overview of the principal working of the model, while section 5 discusses some policy implications. Section 6 provides a summary.

3 Note, however, that only recently the use of robotics and information technology has enabled 'action at a distance' also in the operating theatre. Moreover, Expert Systems embodied in computer programs may in some cases indeed replace a 'live' physician in as far as 'pre-' diagnosing certain illnesses is concerned. Expert Systems may even provide a (hopefully inferior) substitute for psychiatric attention. 


\section{Stylised Models of Endogenous Growth and Health Generation}

From a central planners point of view, both the Lucas (1988) as well as the Romer (1990) model of optimum growth are mathematically equivalent. The Romer model diverges from the Lucas model in two important respects, however. First, growth comes from an increase in the division of tasks which generates efficiency improvements a la Smith. Secondly, decisions are taken by individual agents in situations of imperfect competition, rather than by an all-knowing entity, like it is the case in Lucas (1988). However, we would like to study the allocation of the macro-economic budget from the point of view of the 'planner' (i.e. the government in this case). Hence, we stick to the Lucas model of optimum growth.

Because we want to integrate health and growth in one endogenous growth framework, we also stick to a specification of the production characteristics of the health sector, and its impact on health, which is as simple as possible. To this end, we use the simplifying assumption that the labour force has an infinitely long life, and is constant when measured in physical units.

Instead of concentrating on the provision of health services from an individual demand perspective, as Grossman (1972), followed up by, for instance, Muurinen (1982), Forster (1989) and Ehrlich and Chuma (1990) have done, we will concentrate on the nature of steady state growth equilibria involving the provision of health services and the endogenous generation of knowledge, from a central planners' macro-economic supply perspective. By doing so, we make education and income per head endogenous, instead of taking them to be '... exogenously determined to facilitate an already complex problem' (Ehrlich and Chuma (1990, p. 765)). The decreasing returns to scale character of the health sector, as present in Forster (1989), Ehrlich and Chuma (1990), as well as Johansson and Lofgren (1995), for instance, is retained, while we ignore the difficulties of the timing of the consumption of health services from an individual point of view, by requiring that the average health level of the population of a given size, should remain constant over time. ${ }^{4}$

\section{The Lucas Model of Endogenous Growth}

Optimum growth models have two main ingredients : an accumulation process which can actually cause growth to occur (in Lucas this is the accumulation of knowledge), and some choice criterion which enables the planner to take his decisions only constrained by the production potential of the economy (and changes therein due to growth). Usually one assumes the capacity of production of an economy to be given by a production function which describes the transformation of labour and capital services into output. The role of technical change here is to increase the efficiency of the transformation process. Actually, either technical change is needed in order to counter the fall in the marginal productivity of capital caused by the pure accumulation of capital for given levels of the labour force, or the labour force itself needs to grow in order to avoid capital becoming less and less productive (c.f. Solow (1956)). Hence, capital accumulation alone is not sufficient to generate sustainable growth. The production function, together with the available stock of knowledge and physical capital essentially define the macro-economic budget constraint. The macro-economic budget (i.e. output in this case) can be used for consumption purposes, which generate the utility which society wants to maximise, and for investment purposes. Consumption

4 As a bonus, this makes the analysis more tractable, because a constant steady state average health level for the population, may effectively work in much the same way as the constant allocation of workers to the various productive tasks to be performed within the economy in the Lucas (1988) model. 
increases current utility, while investment increases (potential) future utility by increasing future production capacity and hence future consumption possibilities (but also investment possibilities). Lucas and Romer both assume that societies utility function allows it to trade off future and present consumption possibilities with a constant elasticity of substitution. The above can be summarised as follows :

$$
\begin{aligned}
& U=\int_{0}^{\infty} e^{-\rho t}\left(\frac{(C / L)^{1-\Theta}-1}{1-\Theta}\right) L d t \\
& Y=(A(1-u) L)^{\alpha} K^{1-\alpha} \\
& \frac{d K}{d t}=Y-C \\
& \frac{d A}{d t}=\delta u A
\end{aligned}
$$

where $t=0$ refers to the present. $C, Y$ and $K$ represent consumption, output and the capital stock, respectively. $\rho$ is the rate of discount, while $1 / \Theta$ is the intertemporal elasticity of substitution for consumption possibilities at different points in time. We assume that $\rho>0,0<\Theta \leq 1,0<\alpha<1$, where $\alpha$ is the partial output elasticity of labour. $\mathrm{L}$ is the total amount of labour available in the economy. $\mathrm{L}$ is measured in physical units and is assumed to be constant. $u$ and $(1-u)$ are the fractions of the labour force allocated to the generation of knowledge and output, respectively. $\delta$ is the productivity of the knowledge generation process. A is the per capita knowledge stock, which helps to generate even more knowledge, i.e. increases in the stock make future knowledge generation activities more effective. The production function is Cobb-Douglas, where $\mathrm{A}$ acts as Harrod-neutral technical change. Since the intertemporal utility function is consistent with a constant saving rate, it follows directly from the Solow (1956) model that the steady state rate of growth of the system is equal to the rate of growth of $\mathrm{A}$. By solving (1) through the method of optimum control, one can directly obtain the value of this rate of growth in terms of the parameters of the system ${ }^{5}$ :

$\hat{A}=\frac{1}{\Theta} \cdot(\delta-\rho) \Rightarrow u=\hat{A} / \delta=\frac{1}{\Theta} \cdot\left(1-\frac{\rho}{\delta}\right)$

where a hat over a variable denotes a proportional rate of growth. From (2) it follows that the rate of growth rises with the productivity of the knowledge generation process. It also rises with the value of the intertemporal elasticity of substitution which indicates the willingness of people to wait for their 'consumption' returns on investment (i.e. postponing current consumption till later). Likewise, a rise in the rate of discount indicates a decline in the valuation of future consumption possibilities, and hence reduces the rate of growth of the system. Note that an increased rate of growth also requires an increased allocation of labour to knowledge generation.

5 See Annex A for a derivation. 


\section{A Simple Model of Health Generation}

As observed by Grossman (1976, p. xiii), health contributes in several ways to well-being and economic performance. Especially the positive contribution of a 'good health' to labour productivity is important from a growth perspective. For, this suggests that, in terms of its effects on growth, a change in the average health level of the population works in much the same way as Harrod-neutral technical change in the Solow model or knowledge accumulation in the Lucas model. However, the big difference between knowledge generation and health generation is that, in the end, health generation can not logically be a 'motor' of growth, since health can only continue to grow until a state of perfect health has been attained. ${ }^{6}$ But since a good health is a prerequisite to being able to supply labour services, it still has 'motor-like' features, in the sense that a permanent change in the average health-status of the population could alter growth performance in a permanent way.

In order to elaborate this, let us assume that the average health of the population can be summarised by a one-dimensional index $h$. We furthermore assume that the amount of effective labour services which a person can supply is directly proportional to this index. In addition, it is assumed that the level of health 'deteriorates' instantaneously with an exogenous rate $\zeta$ over time. The task of the health sector then is to counter the effects of this deterioration by providing health services under conditions of decreasing returns to scale, i.e. the average health level of the population rises less than proportionally with the amount of health services per person. Using these assumptions, the evolution over time of the average health level of the population can be written as:

$\frac{d h}{d t}=\delta_{h}\left(\frac{v h L}{L}\right)^{\beta}-\zeta h=\delta_{h}(v h)^{\beta}-\zeta h$

where $\mathrm{v}$ is the share of the health sector in total employment measured in health units, and vhL therefore represents the total input of health units in generating health services. For the sake of simplicity, we assume that the corresponding output of health services is proportional to total inputs. This factor of proportion is subsumed under $\delta_{h}$. Because we assume that the change in the average health status of the population rises less than proportionally with the provision of health services per head, it follows that we require $0<\beta<1$ in equation (3).

Considering the possibility of steady state growth, under conditions of a fixed allocation of human capital over its various productive uses as in the original Lucas model, it is obvious that $h$ should be constant in the steady state instead of continuously growing. However, this is not a very restrictive requirement, since in practice the health level of an individual is bounded from above (and below). The question then becomes what the optimum size of the health sector would be considering the fact that maintaining a certain steady state health level takes scarce resources which generate utility through consumption possibilities.

6 Of course, the latter also holds with regard to knowledge generation. However, contrary to a number of theoretical physicists who believe they can build theories of Everything, we adhere to the idea that the Platonic world of ideas is unbounded on a time-scale which is relevant for forward looking human beings, i.e. a couple of generations rather than aeons. 
The answer is straightforward. First note from (3) that a steady state situation requires $\mathrm{dh} / \mathrm{dt}=0$. Hence, it follows immediately that the steady state sustainable health level, in function of the share of the labour force allocated to the generation of health services, is given by:

$h=\left(\frac{\delta_{h}}{\zeta}\right)^{1 /(1-\beta)} \cdot v^{\beta /(1-\beta)}=z_{0} \cdot v^{z_{1}}$

where $z_{0}$ and $z_{1}$ are implicitly defined by the equivalence of the middle part of (4) and the right-hand side of (4). (4) provides the 'steady state health' production function. (4) shows how the steady state health level depends on the productivity of the health generation process (as reflected by the values of $\beta$ and $\delta_{h}$ ). It also shows that it depends negatively on the rate of decay $\zeta$. Note, moreover that for a health sector with $\beta>0.5$, it follows that $\mathrm{z} 1>1$, i.e. the steady state health production function $\mathrm{h}=\mathrm{z}_{0} \mathrm{v}^{\mathrm{z} 1}$ shows increasing returns, while $z 1<1$ for a health sector with $\beta<0.5 .^{7}(4)$ can now be combined with the Lucas model to describe the optimum investments in health and growth.

\section{On the Contribution of Health to Growth and Welfare}

Health enters the intertemporal decision framework in three different ways. First, a fall in the average health level of the population may be expected to cause a fall in the amount of effective labour services which the population can supply ${ }^{8}$. This points to the complementary character of health and economic performance. Second, the generation of health takes scarce resources which have alternative uses (like the production of output or knowledge), while third, a good health may be expected to influence utility positively. ${ }^{9}$ The latter two points reflect the substitution aspects of health and economic performance.

In the remainder of this section we will first focus on the complementary aspects between health and growth. However, by including knowledge generation a la Lucas, we also cover the second point mentioned above - health is a substitute for growth from

7 Hence, the effective steady state health production function can exhibit increasing returns, even though $0<\beta<1$. We could 'cure' this contradictory situation by means of the 'ad hoc' assumption that the health impact of health services would be inversely proportional to ones health level, in which case (3) could be rewritten as:

$\frac{d h}{d t}=\delta_{h}(v h L /(h L))^{\beta}-\zeta h=\delta_{h} v^{\beta}-\zeta h=0 \Rightarrow h=\frac{\delta_{h}}{\zeta} v^{\beta}$

In this case the effective steady state health production function would exhibit decreasing returns to scale for all values of $0<\beta<1$. However, the qualitative results obtained with the model do not crucially depend on the precise values of $z_{0}$ and $z_{1}$. Hence we stick to the specification of the steady state health production function used in equation (4).

8 Grossman (1972,p. xiii) states : '... the level of ill health, measured by the rates of mortality and morbidity, influences the amount and productivity of labor supplied to an economy'.

9 This is noted in Grossman (1972, p. xiii) : '... what consumers demand when they purchase medical services are not these services per se but rather "good health"'. 
the point of view of health and knowledge generation having to compete for the same scarce (labour) resources. The last part of the section is an extension of the model in order to cover the third point raised above: health will enter the utility function directly.

The Contribution of Health to Growth

As we have discussed above, the task of the health sector is to maintain a certain health level of the population, by providing health services. These are generated under conditions of decreasing returns to scale. Using these notions, the system as presented in (1) can be extended to :

$$
\begin{aligned}
& U=\int_{0}^{\infty} e^{-\rho t}\left(\frac{(C / L)^{1-\Theta}-1}{1-\Theta}\right) \cdot L d t \\
& Y=(A(1-u-v) h L)^{\alpha} K^{1-\alpha} \\
& \frac{d K}{d t}=Y-C
\end{aligned}
$$

$\frac{d A}{d t}=\delta_{A} u h A$

$h=z_{0} v^{z_{1}}$

where the knowledge generation process has the same properties as in Lucas (1988). Note the presence of $\mathrm{h}$ as a determinant of $\mathrm{dA} / \mathrm{dt}$, as well as the absence of $\mathrm{A}$ as a determinant of $h .{ }^{10}$ The latter assumes therefore that A pertains to knowledge which is useful with respect to the generation of output (and further knowledge), rather than the generation of health services.

The system can now readily be solved to obtain: ${ }^{11}$

$g=\hat{A}=\hat{Y}=\hat{C}=\frac{\delta_{A} \cdot(1-\beta) \cdot \bar{h}-\rho}{\Theta}$

Since the first order conditions to the solution of the Hamiltonian problem imply that $v=\beta$, equation (4) implies that the steady state health level $\bar{h}$ is equal to: ${ }^{12}$

$\bar{h}=\left(\frac{\delta_{h}}{\zeta} \cdot \beta^{\beta}\right)^{1 /(1-\beta)}$

10C.f. equations (3) and (4). If h would depend positively on A, and A would be growing in the steady state, then $h$ would be growing too. This can not be permanently the case as suggested earlier.

11 See Annex B.

12 See Annex B. 
where the constraints on the health parameters should be such that $\bar{h} \leq 1$. From (5) it follows that $\bar{h}$ depends positively on the efficiency of the health system and negatively on the rate of decay of health. Note that the amount of labour allocated to the generation of health services depends only on the productivity characteristics of the health sector. Note moreover that at $v=\beta$ the rate of growth of the system as a function of $\mathrm{v}$ is maximised. ${ }^{13}$ This stresses the point that health adds to utility only through its contribution to growth.

Note that the size of the health sector measured either by $\mathrm{v}$ or by $\bar{h}$, depends only on its own characteristics. This is not the case with the rest of the economy, for the fraction of labour allocated to the generation of knowledge can directly be obtained from (6) and the knowledge generation equation as presented in (5):

$u=\hat{A} /\left(\delta_{A} \bar{h}\right)=\frac{(1-\beta)}{\Theta}-\frac{\rho}{\Theta \delta_{A} \bar{h}}$

Hence, growth and the allocation of labour among the non-health sectors do not depend exclusively on the productivity characteristics of these sectors. Instead, their performance depends intrinsically on the characteristics of the health sector.

Equations (6) and (8) are comparable to the results obtained in the basic Lucas model (cf. equation (2)). When we would assume that the parameters of the health-system are such that $\bar{h}=1,{ }^{14}$ the only difference between the Lucas growth rate and $g$ in equation (6) is the term $(1-\beta)$, which reflects the fact that a fraction $\beta$ of the labour force is not available for the generation of output and knowledge since its task is to maintain the average health level of the population at its steady state value $\bar{h}$. This lowers the maximum rate of growth attainable compared to the Lucas model.

The Contribution of Health to Welfare

Up to now we have ignored the welfare aspects of a good health. However, we can include these in our model by introducing the average health level $h$ as a direct determinant of society's utility, where a higher value of h corresponds to a higher level of utility. We can do this by replacing consumption per head in the utility function by a term which incorporates the average health level in the following way:

$U=\int_{0}^{\infty} e^{-\rho t}\left\{\frac{\left((h / \bar{h})^{\gamma}(C / L)\right)^{1-\Theta}-1}{1-\Theta}\right\} \cdot L d t$

13 This follows directly from maximising (B.16) in Annex B with respect to v.

14 In this case the Lucas model and our model are equivalent from the point of view of the productivity of the labour force in its non-health uses. 
where we assume $\gamma \geq 0 .{ }^{15}$ The average health level is measured relative to the steady state health level of the previous model, so that, when $\gamma=0$ or close to zero, both models would generate more or less the same results. This makes both models more comparable.

The consequences of health entering the utility function can not readily be determined in an analytical fashion, because the first order conditions to the solution of the Hamiltonian system including (9) give rise to the following non-linear reduced form system in $\mathrm{h}, \mathrm{v}, \mathrm{g}$, the average propensity to consume $\mathrm{c}=\mathrm{C} / \mathrm{Y}=1-\mathrm{s}$ and $\mathrm{u}:{ }^{16}$

$f=c^{2}-\alpha \cdot c$

$v=\frac{f+\alpha \cdot(1-\alpha) / \gamma}{f+(\alpha / \beta) \cdot(1-\alpha) / \gamma]}$

$g=\left(\delta_{A} \cdot(1-v) \cdot z_{0} \cdot v^{z 1}-\rho\right) / \Theta$

$c=1-\frac{(1-\alpha)}{\Theta+\rho / g}$

$u=(1-c) \cdot(1-v) /(1-\alpha)$

Note from equation (10.E) that in order for $1-u-v>0$, it is necessary that $\alpha<c<1 .{ }^{17}$ Hence, this restriction on $c$ would imply that $u$ is positive and that some output would indeed be produced in the steady state. In addition, it implies that the steady state saving rate will always have to be smaller than $1-\alpha$.

Once the simultaneous solution of equations (10.A-10.D) has been found, the solution of (10.E) can be obtained directly. ${ }^{18}$ However, (10.A-10.D) can not be solved in a straightforward way. But (10.A-10.D) can be represented as a four quadrant diagram, which enables us to use a graphical analysis instead. ${ }^{19}$ Even without an extensive analysis of this system, we can see that the introduction of health as an argument in the utility function links growth directly to the characteristics of the health sector as it

15 This specification is consistent with the one suggested by Barro and Sala-i-Martin (1995, p. 323) in their discussion of working time entering the intertemporal utility function as a negatively valued item, with ever increasing marginal dis-utility. In our case we have the average health level of a person entering the utility function in a positive way. The marginal utility of health will be decreasing when $\gamma<1$.

16 See Annex C for more details.

17 This requirement is consistent with the Kaldorian idea of differential saving, where workers consume all of their income and where we require capitalists to consume at least some of their income.

18 Note that when $v=\beta$, as in the model without health as an argument of the intertemporal utility function, the simultaneity of (10.A)-(10.D) disappears, since $\mathrm{g}$ and $\mathrm{c}$ follow recursively from $v$. Hence, the health sector determines growth performance, but health is determined independently from growth.

19 The mapping procedure which underlies this approach is described in more detail in Annex D. 
was the case in the first model. But the direct contribution of health to welfare now also links the size of the health sector to the characteristics of the rest of the economy. This is shown in more detail below.

\section{A Graphical Analysis}

Equations (10.A), (10.B) and (10.D) imply a relationship between $g$ and $v$, which can be confronted with (10.C). This is elaborated in Figures 1 and 2.

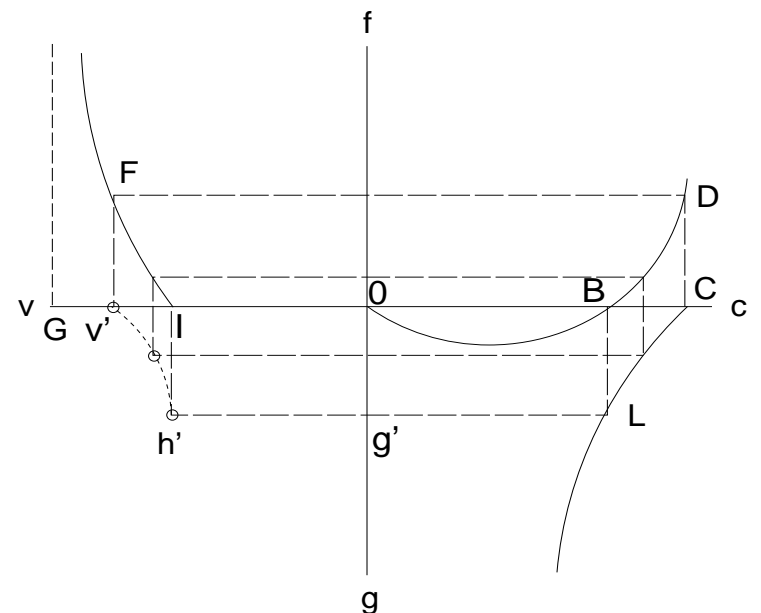

Figure 1

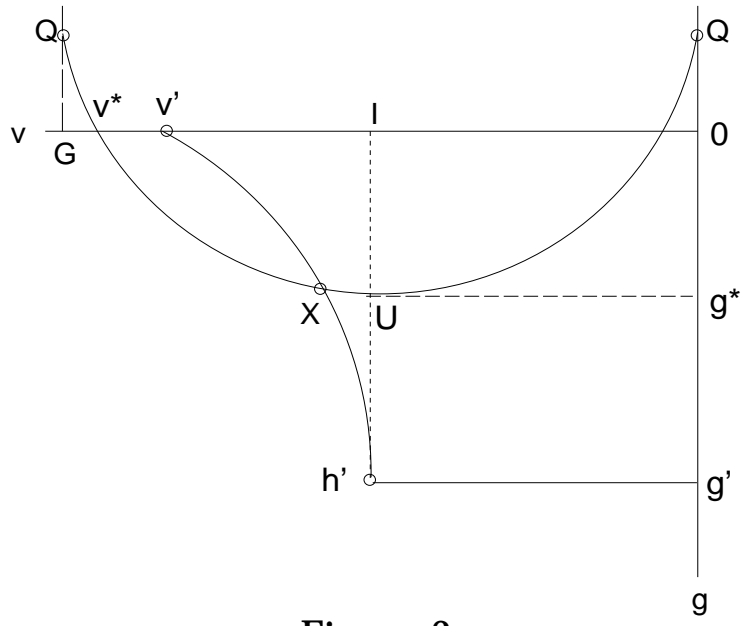

Figure 2

In Figure 1, $\mathrm{c}$ is the average propensity to consume, while $f(c)=c .(c-\alpha)$ is a parabola in $c$ with roots $c=0$ and $c=\alpha$. v represents the fraction of labour employed in the health sector, while $\mathrm{g}$ is the rate of growth of the system.

Equation (10.D) implies the existence of two extreme values for $c$, i.e. $c=1$ for $g=0$, marked as point $C$ in Figure 1, and $c=1-(1-\alpha) / \Theta$ for $g$ goes to infinity. The point B corresponds to $c=\alpha$, hence the curve CL represents the relevant part of equation (10.D). In the same vein, BD is the relevant part of (10.A). Equation (10.B) implies that $v=\beta$ for $f=0$, while for $f$ goes to infinity, $v$ would asymptotically approach a value of 1 . That is, point $\mathrm{G}$ in Figure 1 corresponds with $\mathrm{v}=1$, while point I corresponds with $v=\beta$. The relevant part of equation (10.B) is the curve IF. The relevant range for $v$ is $\beta \leq v \leq v$, while the relevant range for $g$ is $0 \leq g \leq g,{ }^{20}$ Any point within the latter range corresponds with a unique point in the former range by mapping $g$ onto $c$, c onto $f$ and then f onto $v .^{21}$ Hence, the curve $v^{\prime} h^{\prime}$ in Figure 1 summarises equations (10.A), (10.B) and (10.D). The curve $v^{\prime} h^{\prime}$ can then be confronted with (10.C) to obtain the solution of the model.

Equation (10.C) is represented in Figure 2. Figure 2 corresponds to the South-West quadrant of Figure 1. It has the same orientation as in Figure 1. As in the solution of the previous model, $g(v)$ reaches a maximum for $v=\beta$, i.e. in point $U$ (see equations (6) and (10.C)). The points of intersection $Q$ with the vertical axes in $v=1$ and $v=0$

20 Note that $g^{\prime}=\rho /(1-\Theta)$, while $v^{\prime}=\beta(\gamma+\alpha) /(\beta \gamma+\alpha)$.

21 By 'going round' in Figure 1 in a counter-clockwise direction, it follows that point $\mathrm{g}^{\prime}$ on the g-axis, for instance, corresponds to point I on the v-axis through the connections between the 'intermediate' points $\mathrm{L}, \mathrm{B}$, and 0 . Likewise, point $\mathrm{g}=0$ on the $\mathrm{g}$-axis maps onto points $\mathrm{C}, \mathrm{D}, \mathrm{F}$ and finally point $\mathrm{v}^{\prime}$ on the $\mathrm{v}$-axis. 
correspond to a value for $g$ of $g=-\rho / \Theta$ (see (10.C)). For values of $v$ such that $v>\beta$, the curve $\mathrm{g}(\mathrm{v})$ is concave and it describes the negative relation between $\mathrm{g}$ and $\mathrm{v}$ for $v>\beta$. The value of the growth rate corresponding to $v=\beta$ is $\mathrm{g}=\mathrm{g}^{*}$, while $\mathrm{g}=0$ at $\mathrm{v}=\mathrm{v}^{*}$. ${ }^{22}$ The relevant part of equation (10.D) is the curve $U v^{*}$. The relevant range for $v$ is $\beta \leq v \leq v^{*}$, while the relevant range for $\mathrm{g}$ is $0 \leq g \leq g^{*}$.

The solution of the model is the point of intersection $X$ between the curves $U v^{*}$ and $v^{\prime} h^{\prime}$. A unique solution exists when the curve $v^{\prime} h^{\prime}$ is convex and when $g^{\prime}>g^{*}, v^{*}>v^{\prime}{ }^{23}$ The requirements for convexity of the curve $\mathrm{v}^{\prime} \mathrm{h}^{\prime}$ are elaborated in Annex $\mathrm{E}$, where it is shown that for plausible values of the parameters of the system, these constraints are likely to be satisfied. ${ }^{24}$

From Figure 2 it is clear that the point of intersection between the two curves implies a value of $\mathrm{g}$ which is lower than in the first model. Moreover, $\mathrm{v}$ is larger than before. Hence, the introduction of health in the utility function increases the level of health services at the expense of growth, ceteris paribus. A more detailed analysis of the properties of the model is presented in the following section.

\section{Parameter Changes and Health and Growth Responses}

In order to illustrate what happens to health, growth and to the size of the health-sector for various constellations of the parameters of our model, we have used a graphical analysis based on Figure 2. The technical details of the analysis are presented in Annex F. In this section we just summarise the results in Table 1 below. Positive and negative influences of a (positive) change in a parameter are indicated by a plus and a minus sign, respectively. Ambiguous reactions are indicated by a question mark followed by a sign within brackets which indicates a relatively high probability of the effect having that particular sign.

The first thing to be noted from this table is the negative correlation between the effects of a parameter change on growth and on the propensity to consume. This is a consequence of the fact that there is a positive correlation between the saving rate $s$ and the rate of growth itself, since, for a relatively stable value of the capital output ratio, a rise in the saving rate would be required in order to speed up the accumulation of capital which is necessary in order to sustain a higher rate of growth. Secondly, $v$ and $u$ are negatively correlated, instead of positively as suggested by Fuchs (1982), for instance.

22 The way in which $\mathrm{g}^{*}$ and $\mathrm{v}^{*}$ depend on the parameters of the model can be summarised by $g=g *\left(\delta_{A}(+), z_{0}(+), \beta(+), \rho(-), \Theta(-)\right)$ and $v=v^{*}\left(\delta_{A}(+), z_{0}(+), \beta(+), \rho(-)\right)$, where the sign within brackets denotes the sign of the partial derivative with respect to the parameter in question. These results follow directly from equation (10.C) and the requirement that $g\left(\mathrm{v}^{*}\right)=0$.

23 In Annex $\mathrm{D}$ we show that these are sufficient conditions for the mapping process to converge to a solution with $\alpha<c<1$, which was required to ensure a positive level of output.

24 If $v^{\prime} h^{\prime}$ would be concave, then, in principle at least, there could be three points of intersection between the curves $U v^{*}$ and $v^{\prime} h^{\prime}$ even with the constraints on the shape of $v^{\prime} h$ that we have used. But even if $v^{\prime} h$ ' would be concave, as long as it defines a unique point of intersection between $\mathrm{v}=\mathrm{z}(\mathrm{g})$ and $\mathrm{g}=\mathrm{g}(\mathrm{v})$, the way the system works will not be affected by its curvature. In that case only the intensity of the responses of the system to parameter changes will be affected. In order to simplify matters, however, we will assume that parameter constraints are such that $v^{\prime} h^{\prime}$ is convex. 


\begin{tabular}{|c|c|c|c|c|c|}
\hline Param & $\mathrm{c}=1-\mathrm{s}$ & $\mathrm{v}$ & $\mathrm{g}$ & $\mathrm{u}$ & $\mathrm{h}$ \\
\hline$\Theta$ & + & + & - & - & + \\
\hline$\rho$ & + & + & - & - & + \\
\hline$\delta_{A}$ & - & - & + & + & - \\
\hline$\delta_{H}$ & - & - & + & + & + \\
\hline$\zeta$ & + & + & - & - & - \\
\hline$\gamma$ & + & + & - & - & + \\
\hline$\beta$ & $?(-)$ & + & $?(+)$ & $?(+)$ & + \\
\hline
\end{tabular}

Table 1 Parameter Changes and Health and Growth Responses

A possible explanation for the latter positive correlation lies in the fact that increases in health investment would lengthen one's life-span, ceteris paribus, and hence increase the returns on investment in education. An alternative explanation would be that a higher level of education would go hand in hand with increases in the preference for health (possibly due to the rise in the opportunity costs of not being healthy). In our model the negative correlation exists because both health and knowledge generation compete for the same scarce labour resources, while the fixed size of the labour force in physical units doesn't allow for endogenous variations in life-span.

Let us now turn to the individual results. A rise in $\Theta$ implies a fall in the value of the intertemporal elasticity of substitution. This means that people become more reluctant to wait for their return on investment, and consequently they are inclined to increase current consumption of goods, but also of health services. This is reflected in a rise in the steady state values of $\mathrm{c}, \mathrm{v}$ and $\mathrm{h}$, accompanied by a fall in $\mathrm{g}$ itself, but also in $\mathrm{u}$.

The results for a rise in $\Theta$ are very similar to those obtained for a rise in the rate of discount $\rho .^{25}$ This is logical since a rise in $\rho$ reflects the decrease in the subjective valuation of the utility derived from the consumption of a certain package of goods and health-services in the future relative to the valuation of the utility of that same package when it would be consumed today. Hence, one would expect people to spend more resources on fulfilling current needs, by redirecting labour input to activities which increase present consumption possibilities, rather than future ones. Consequently, c, v and $\mathrm{h}$ rise, while $\mathrm{g}$ and $\mathrm{u}$ fall.

A rise in the productivity of the knowledge generation sector, i.e. $\delta_{A}$, has quite different effects. Since the latter increases the marginal benefits of investing in knowledge generation, a re-allocation of labour from activities which increase current utility to activities which increase future utility can be observed. As a result, growth is positively affected. In order to make this possible, $c$ and $v$ are reduced, while $u$ and $g$ are increased. $\mathrm{h}$ falls because of the reduction in $\mathrm{v}$. Note that these results are very similar to those obtained for an increase in the productivity of the health sector, i.e. $\delta_{h}$, which corroborates the 'motor-like' features of the health sector mentioned in section 2 of this paper. Because an increase in $\delta_{h}$ would tend to increase $\mathrm{h}$ for a given $\mathrm{v}$, it would also, and

25 There are differences though, as explained in more detail in Annex F. 
permanently, increase the productivity of the knowledge generation process. The productivity increase in the health sector then enables a contraction of the allocation of labour resources to that sector (v falls), while h rises, which in turn makes investment in knowledge generation more profitable because of its intertemporal spill-over effects. Hence $u$ rises, and so does $g$.

The effects of a rise in the rate of decay on health is somewhat ambiguous, according to our graphical analysis, because a rise in $\zeta$ would tend to lower the average health of the population. This by itself would reduce the productivity of the knowledge generation process, which would tend to reduce $u$, as explained above. The reduction in the grow th rate which this entails enables an increase in the propensity to consume (c rises). However, in order to counter the negative growth effects of a rise in $\zeta$, the health sector expands ( $v$ rises), but not enough to sustain the original level of health due to the decreasing returns to scale character of the health sector. ${ }^{26}$

The effects of a rise in the preference for health, i.e. $\gamma$, are completely as expected. More labour resources are drawn into the health sector ( $v$ rises), while both growth itself and the allocation of labour to knowledge generation $(\mathrm{u})$ are reduced. The fall in the rate of growth enables a reduction in the saving rate, and hence a rise in c, while $h$ rises too, as one would expect.

Finally, a rise in $\beta$ has ambiguous effects. ${ }^{27}$ The reason is that a change in $\beta$ has immediate consequences for the orientation of the 'curve' $v=v(f)$ in Figure 1. It also has profound consequences for the steady state growth path as given by equation (6): the 'top' of $\mathrm{g}=\mathrm{g}(\mathrm{v})$ is reached for a higher value of $\mathrm{v}$ (recall that $\mathrm{g}(\mathrm{v})$ reaches a maximum for $v=\beta$ ), while both $z_{0}$ and especially $z_{1}$ are positively affected. So $\mathrm{v}$ is increased because of the increase in the overall productivity of the health sector, while growth performance itself as given by equation (6) is also positively affected by a rise in $\beta$. However, an increase in $\mathrm{v}$ reduces growth, ceteris paribus, and hence the overall impact of a rise of $\beta$ on growth would be ambiguous. Nonetheless, one would expect that the rise in the marginal productivity of a health worker due to the increase in $\beta$, would not result in

26 In the decreasing returns to scale setting we have defined, one would expect a reaction to an exogenous shock not to be able to wipe out all the effects of such a shock, because such a reaction would involve the re-allocation of resources which were initially allocated in such a way as to generate maximum overall benefits for a given stock of scarce irreproducible labour resources. In fact that is exactly what we found during exploratory simulations with our model using 'reasonable' parameter values, where we observed a fall in $h$ despite the rise in $\mathrm{v}$ which is required to counter the effects of the rise in $\zeta$.

27 As stated earlier, the decreasing returns character of the health sector lies in part in the decrease in the marginal health benefits of the consumption of health services from the individuals point of view. If that is a 'technical datum', then $\beta$ can of course not be altered. However, from the point of view of the health sector and the population as a whole, one could imagine being able to increase $\beta$ by abstaining from supplying services which are needed by just a minor fraction of the population, and which take a relatively, large share of total resources. Roughly speaking, this amounts to giving up 'hopeless' cases and spending the resources on more 'promising' cases. Of course, we do not take that stance, since this raises very important questions which can not really be addressed here, if only because of the simple specification of the utility function used in our model. Consequently, for our purposes we take $\beta$ to be a 'technical datum'. 
an expansion of $\mathrm{v}$ such that in the end the marginal productivity of a health worker will have fallen, the more so because the increase in $\mathrm{v}$ can only be brought about by reducing the allocation of labour to other activities at increasing opportunity costs.

\section{Policy Relevance : Some Illustrative Simulations}

In this section we present a number of simulations which we have run in order to illustrate the possible relevance of the model for very diverse situations, ranging from a Baumols disease like diagnosis for the productivity slow down experienced in the West, to the effectiveness of technology transfer or aid in general in speeding up the pace of welfare improvements in the less developed countries. The potential relevance for these situations follows directly from a number of implications of the model.

First of all, the fact that we have a decreasing returns health sector which level of activity defines the effective availability and applicability of knowledge within the economy, makes the efficiency of this sector one of the central determinants of economic performance. Indeed, the notion that effective inputs of human capital and labour into the various production processes depend on ones health status, makes health a complement to growth from a supply perspective. Moreover, a change in $\delta_{h}$ may have far more important effects on growth than an equal proportionate change in $\delta_{A}$, for a health sector with moderate decreasing returns to scale features. ${ }^{28}$ This stresses the importance of health as a determinant of both the level and the growth of labour productivity, quite apart from the direct positive welfare effects which changes in the productivity of the health sector may entail, when a good health as such would be positively valued.

Secondly, the influence of the decreasing returns to scale nature of the health sector on growth, provides an interesting alternative explanation for the productivity slowdown. If, as seems reasonable to assume, the preference of people for a good health rises with the standard of living, i.e. $\gamma$ rises with output per head, then growth would automatically slow down in the process. Thus the decreasing returns to scale nature of the health-sector may be regarded as a manifestation of Baumols disease in a dynamic context.

Third, this dynamic Baumols disease notion becomes the more relevant, in the face of the fact that the average age of the population in Western European economies has shown a tendency to rise during the last decades. This introduces a wedge between the two functions of the population in our model. On the one hand the population provides the scale of available labour supply, and hence the scale of all economic activities which rely on the use of labour services. On the other hand, the size of the population also determines the scale of the demand for health-services. Hence, a rising trend in the average age of the population, for a given total size of the population, could be expected to provide an additional break on productivity growth.

28 This follows directly from equations (4) and (10.C). The latter equation shows that $z_{0}$ and $\delta_{A}$ influence the growth rate in exactly the same way. However, from equation (4) it follows that $\hat{z}_{0}=\frac{1}{1-\beta} \cdot\left(\hat{\delta}_{h}-\xi\right)$. So, the initial impact on growth of a change in $\delta_{h}$, is larger, ceteris paribus, than an equal proportionate change in $\delta_{A}$. 
Fourth, in the case of high values of the rate of decay of health, due to malnutrition for instance, people may have such a high preference for consumption now, that they could become stuck in a 'no growth', 'low health' trap. ${ }^{29}$ With a (near) zero preference for health, we would have $v=\beta$, i.e. the health level of the population would be constant, as long as nothing would change in the 'health parameters' $\zeta$ and $\delta_{h}$. This suggests that policies aimed at furthering growth by means of reducing $\zeta$, or increasing $\delta_{h}$, through direct aid in the form of technology or income transfer, may induce growth which is sufficiently high to lower the rate of discount $\rho$ and increase the intertemporal elasticity of substitution $1 / \Theta$ to such an extent, that savings will arise which allow growth to take off and become self-sustaining.

\section{Simulation Results}

The remainder of this section shows the outcomes of some simulation experiments which we have run to highlight the points raised above. To this end, we have defined a computer version of the model which encompasses equations (10.A-10.E) ${ }^{30}$, which describe steady state growth behaviour with respect to the allocation of labour resources over their various uses as well as the steady state propensity to consume. We have fed the values of these variables into the knowledge accumulation equation, as well as the capital accumulation equation to arrive at adjustment paths for the level of output, (physical and knowledge) capital, and health and income per head. ${ }^{31}$ Then output is generated in accordance with the Cobb-Douglas production function. We have calibrated the initial value of the physical capital stock in such a way, that the growth rate generated for this initial value of the capital stock in combination with the steady state propensity to save, was equal to the steady state growth rate $g$ itself, implicitly defined by equations (10.A-10.E). ${ }^{32}$ The initial stocks are denoted by a subscript zero. The parameter values and the initial values which we have used to generate the base run are given in Table 2 below.

The parameters have been chosen in such a way that the model generated 'reasonable' values of the steady state growth rate, the steady state health level, etcetera. The outcomes of the model based on these parameter values are summarised in Table 3 below. The outcomes were obtained for $\gamma=0.5$.

29 In terms of the features of the model this would mean that $\zeta, \rho$ and $\Theta$ would be large, while $\gamma$ would approach zero. This would push the $g=g(v)$ curve in Figure 2 up, while $\mathrm{v}$ would be equal to $\beta$ as it was the case in our first model where health was just a complement to growth.

30 See Annex $G$ for a complete model listing.

31 Note that these do not have to be the optimum paths for the variables under consideration. We use these paths to illustrate that they have a strong tendency to converge rapidly to the steady state growth path.

32 The reason to do so is that we want to see how the state variables will adjust themselves after a change in the parameters or the exogenous variables of the model. This allows us to see whether, and if so, how fast the growth rates of the state variables will reach their 'new' steady state values as described by (10.A-10.E). 


\begin{tabular}{|c|c|c|c|c|c|}
\hline Param & Value & Param & Value & Param & Value \\
\hline$\alpha$ & 0.650 & $\delta_{A}$ & 0.114 & $\beta$ & 0.125 \\
\hline$\rho$ & 0.075 & $\delta_{h}$ & 0.059 & $K_{0}$ & 4.778 \\
\hline$\Theta$ & 0.500 & $\zeta$ & 0.050 & $L, A_{0}$ & 1.000 \\
\hline
\end{tabular}

Table 2 Base Run Parameter Values

\begin{tabular}{|c|c|c|c|c|c|}
\hline Variable & Value & Variable & Value & Variable & Value \\
\hline $\mathrm{c}$ & 0.898 & $\mathrm{~h}$ & 0.936 & $\mathrm{z}_{0}$ & 1.200 \\
\hline $\mathrm{g}$ & 0.026 & $\mathrm{u}$ & 0.240 & $\mathrm{z}_{1}$ & 0.143 \\
\hline$\gamma$ & 0.500 & $\mathrm{v}$ & 0.175 & - & - \\
\hline
\end{tabular}

Table 3 Steady State Values Model-Variables

In the remainder of this section, we present the graphs pertaining to the simulation results we have obtained for a number of experiments meant to cover the 4 points raised above. The results are based on simulations from time 1 up to time 300 . We have chosen a long simulation period in order to be able to see whether, and if so, how fast the model variables would approach their steady state values.

Income Dependent Preferences for Health and an Ageing Population

We have specified a Lorentz-curve like linkage between the preference for a good health $\gamma$ and income per head $y$ :

$\Delta \gamma=\varepsilon \cdot(1-\gamma) \cdot \max (0,1-\bar{y} / y)$

where $\bar{y}$ denotes minimum income per head and where we have set $\bar{y}=1.45$. $\varepsilon$ has been set equal to $0.05 . \gamma$ starts rising as soon as $y>\bar{y}$, while the maximum value which $\gamma$ can attain has been set equal to 1 .

In order to show the separate influences of the dependence of health preferences on income per head and of an ageing population on the growth performance of the economy, we assume that this ageing occurs from period 200 up to 300 . A rise in the average age of the population can be 'simulated' by assuming that the part of the population that is actively engaged in producing output, knowledge and health, decreases relative to the total population. More in particular, from period 200 up to period 300, we let the size of the active population fall by 5 percent, whereas the total population remains at a value of 1 . We would expect this to lead to a decrease in growth, because the productivity of the health sector, i.e. $z_{0}$, is negatively affected by a decrease in the ratio of active people versus inactive people. This follows immediately from equation (3), which can be rewritten as:

$\frac{d h}{d t}=\delta_{h}\left(\frac{v h \psi L}{L}\right)^{\beta}-\zeta h=0 \Rightarrow h=\psi^{\beta /(1-\beta)} z_{0} v^{z_{1}}=z_{0} v^{z_{1}}$ 
where $\psi$ denotes the share of active people in the total population $\mathrm{L}$, and $\mathrm{z}_{0}{ }^{\prime}$ is implicitly defined by (12). Since we let $\psi<1$, it follows that the effective productivity of the health sector, i.e. $\mathrm{z}_{0}{ }^{\prime}$, is negatively affected, in the sense that it becomes more difficult to maintain a constant average health level of the total population for a declining share of active people.

The simulation results of this experiment, denoted by the prefix $\mathrm{X} 1_{-}$, are presented in Figures 3-7. The sigmoid character of the adjustment in $\gamma$ is immediately clear from Figure 3 : we see $\gamma$ rising from a value of zero to a value of one in roughly 200 periods. In Figure 4, we present the steady state growth rate $(\mathrm{G})$ as well as the actual growth rate of the capital stock (G_K), when $c$, $u$ and $v$ are adjusted in accordance with steady state optimum behaviour, as given by equations (10.A-10.E). In the base run, denoted by the prefix $X 0_{-}$, the actual growth rate of $\mathrm{K}$ is equal to the steady state growth rate. That is due to the calibration exercise mentioned earlier. We also see that the increase in $\gamma$ does indeed lead to slower growth, as suggested above. Moreover, we see that changes in the actual growth rate lag somewhat behind the changes in the steady state growth rate, but not by more than $5 / 100$ of a percentage point. We see that a doubling of $\gamma$, i.e. $\gamma$ rising from a value of 0.5 in the base run to a value of 1 in experiment 1 , leads to a permanent fall in the steady state growth rate of about 0.5 percentage points. Note too, that the propensity to consume is increased (Figure 5), whereas the average health level increases as well (Figure 6), at least while $\gamma$ is rising. In Figure 7, we see that the increased preference for health diminishes the incentive to invest in knowledge generation ( $u$ falls). However, all of the fall of $u$ is absorbed by the health sector, since $u+v$, denoted by U_PLUS_V is hardly changed at all. We conclude therefore that a rise in the preference for health leads to slower growth and to a re-allocation of resources in the direction of the health sector. We conclude that if $\gamma$ is indeed positively influenced by income per head, then the model predicts the occurrence of dynamic 'Baumols disease-like" phenomena for countries with a growing income per head.

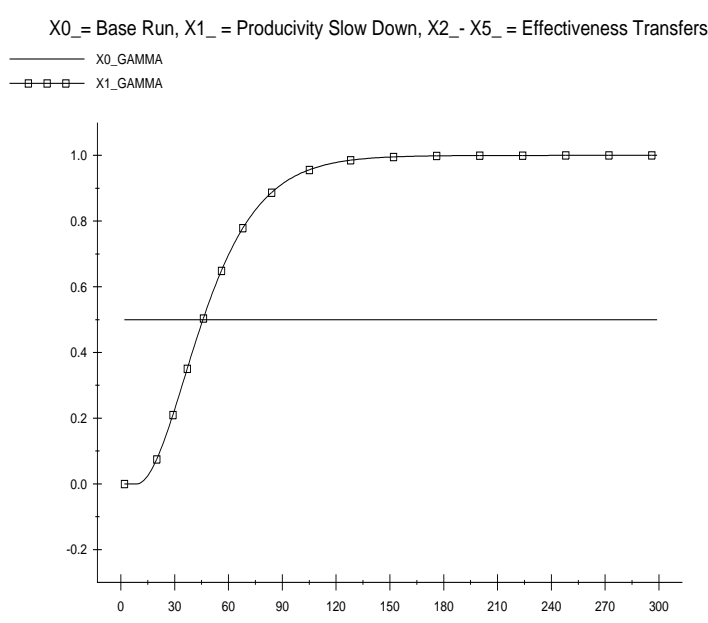

Figure 3

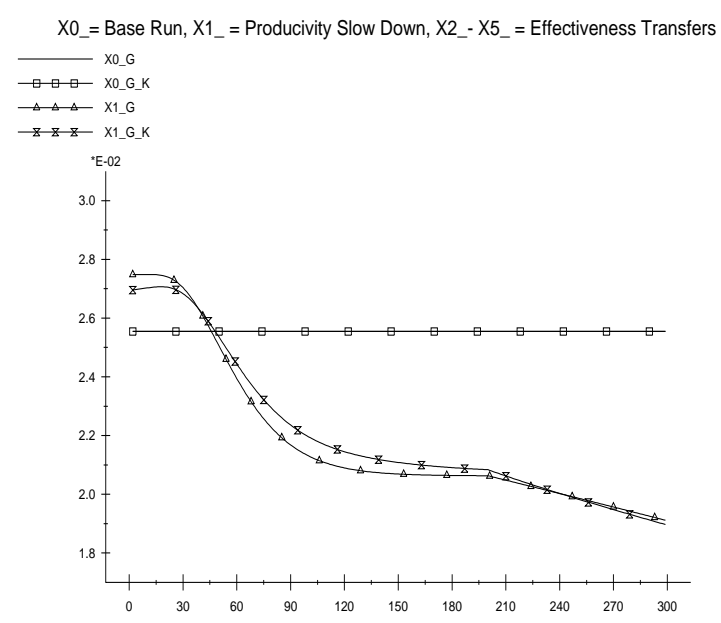

Figure 4 


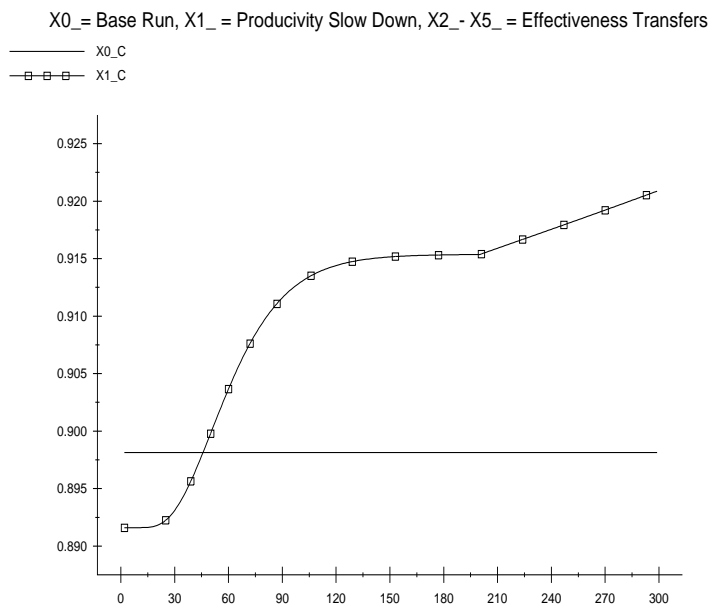

Figure 5

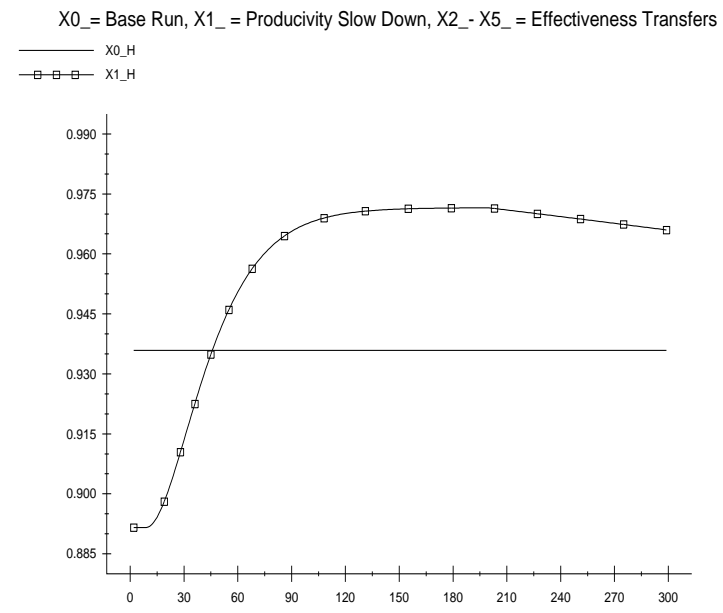

Figure 6

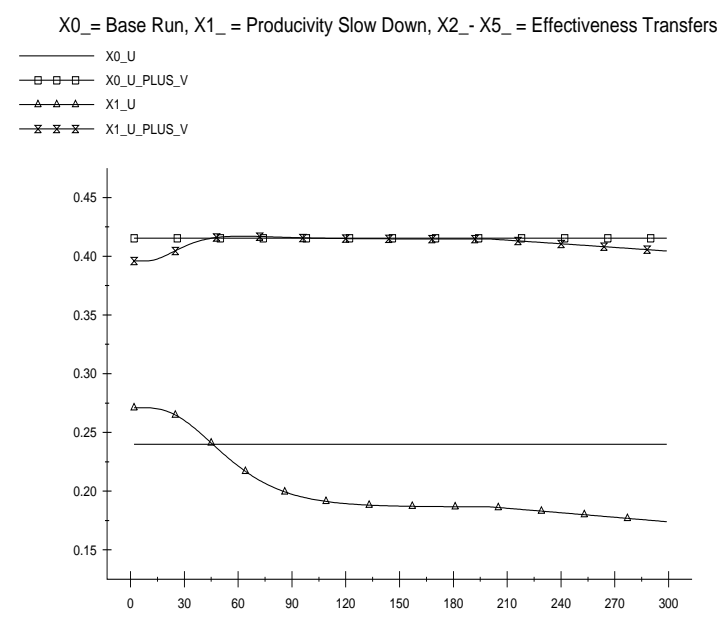

Figure 7

The fall in the share of active people in the population is taking place from period 200 onwards, for a stable value of $\gamma$ which from period 200 onwards is equal to one. Hence, all the ensuing changes in growth performance can be attributed to the change in the composition of the population. Note that in this part of experiment 1, the 'health demand base' of the economy remains the same, while available scarce productive labour resources diminish in number. Because of the implied decrease in the effective productivity of the health sector $\left(\mathrm{z}_{0}{ }^{\prime}\right)$, and the negative consequences this has for the steady state health level of the population (and therefore for growth itself, see Figure 6 which shows a slight fall in $h$ after period 200), labour resources are re-allocated between knowledge generation, output generation and health production : $u$ falls from a value of 0.187 in period 200 to a value of 0.174 in period 300 , while $v$ rises slightly from a value of 0.228 to a value of 0.231 during the same period. Consequently, $u+v$ falls slightly from a value of 0.415 to a value of 0.405 , which implies a slight increase in the share of active people allocated to output generation. The reason why this happens, is that the knowledge generating sector experiences a drop in the growth rate of its productivity, which adds to the initial fall in its productivity level induced by the decline in average health levels. By contrast, the direct effect of the initial decline in average health is confined to just the level of productivity in the output producing sector. Consequently, the marginal benefits of allocating additional labour to knowledge production decline more than the marginal benefits of the allocation of labour to 
the other productive activities, including the generation of health services. Our main conclusion is then that because of the negative effects on productivity growth in knowledge generation, the system shows a tendency to concentrate its efforts on mitigating the 'blow' to growth caused by a fall in average health, rather than on compensating the fall in productivity growth by increasing knowledge generation efforts. The net effect is nonetheless that the growth rate falls, despite the counteracting movements in v (see Figures 6 and 7), and c can rise as a result (see Figure 5).

\section{Aid as a Means to Provide Growth and Health}

We have also run a number of simulation experiments to highlight the fourth point raised above. More in particular, we have assumed here that the model now describes a country which is 'poor' and caught in a 'low health', 'no growth' trap ${ }^{33}$ because it has such a high rate of discount, that its $g=g(v)$ curve lies almost entirely above the horizontal v-axis in Figure 2, i.e. maximum growth is equal to zero. In addition, we implement this no growth trap by assuming that the propensity to consume can only fall below a value of unity when income per head is higher than a certain minimum value : when income per head would be below that value the country is simply too poor to save, but just not poor enough for the population to starve.

Given this design of the experiment, there are a number of ways in which the welfare of the country caught in the 'low health', 'no growth' trap could be raised. For example, the rate of decay of health $\zeta$ could be lowered either directly through medical aid, or indirectly through improving general living conditions. This reduction in $\zeta$ would raise $\mathrm{z}_{0}$ (c.f. equation (4)), and hence this would raise the effective productivity of the health sector, which in turn would enhance growth. We have run an experiment to this effect, which we do not show here, in which health rises by more than the percentage decrease in $z_{0}$, because the increased productivity of the health sector makes an expansion of the level of health-services more profitable relative to other uses of knowledge, especially with regard to the production of output. In this experiment, investment in knowledge generation is also positively affected, due to the impact of the increase in average health levels on the growth rate of productivity in the knowledge producing sector.

Another form of aid could be envisaged, i.e. the transfer of 'income', such that income per head in the receiving country rises instantaneously to a certain percentage $\pi$ above minimum income per head $\bar{y}$ (i.e. the level which is just sufficient to maintain a stable health level of the population at a zero level of savings). In this case, the amount of income transferred is given by equation (13.A), while total available income is given by equation (13.B):

$$
\begin{aligned}
& T=\max (0,(1+\pi) \cdot \bar{y} \cdot L-Y) \\
& Y=((1-u-v) h A L)^{\alpha} K^{1-\alpha}+T
\end{aligned}
$$

In addition to these income transfers, we also let the rate of discount fall with a rise in income per head, because a higher standard of living enables people to become more forward looking both in principle and in practice. Income is transferred whenever and as long as actual income per head lies below the minimum level $\bar{y}$.

33 Note that for these experiments we have only changed the rate of discount in such a way that $g(\beta)=0$. Hence, strictly speaking, the 'low health' characterisation does not really apply here. 
Various experiments with this set up have shown us that there is a very narrowly defined value of the transfer-rate $\pi$ for which the transfer of income starts to work, in the sense that saving rates in the receiving countries can rise, thus enabling the accumulation of capital. These experiments also show that too little aid may cause growth never to take off, while too much aid may just increase the bill for all parties concerned without generating any significant additional growth and health benefits at the receiving end. Note that the results summarised in Table 1 would indicate that a fall in $\rho$ as induced by a rise in income per head, would actually tend to lower $\mathrm{v}$, and hence $h$, although growth itself would increase. However, it stands to reason that the preference for health as one of the most important basic human needs next to the need for food and shelter, would rise so fast with income per head, that the increased opportunity costs of the provision of health in comparison with knowledge generation, is more than outweighed by the increase in $\gamma$, so that the net effect on $h$ would tend to be positive. The results of the various experiments are presented in Figures 8-14. In these Figures the prefixes $\mathrm{X} 2_{-}, \mathrm{X} 3_{-}, \mathrm{X} 4{ }_{-}$and $\mathrm{X} 5$ _ are associated with different values of the transfer rate $\pi$, i.e. $0.0051,0.0052,0.01,0.02$ respectively. Note that in this series of experiments we have started with $\gamma$ growing from its base run value of 0.5 and with $\varepsilon=0.025$, which implies a slower adjustment of the preference for health to income per head, but $\gamma$ starts changing from a higher level than in experiment 1.

The possibility that growth may never take off despite the transfer of income, is reflected in Figure 8, which shows that in experiment 2 nothing happens, while experiments 3-5 all show similar developments of the preference for health. This indicates that, at least in experiments 3-5, the pace of development of income per head is similar too, despite the fact that the transfer rate in experiment 5 is about four and two times as large as the transfer rates in experiments 3 and 4, respectively. Note that it is possible to escape from the no-growth trap by raising $\pi$ ever so slightly from a value of 0.0051 to a value of 0.0052 , as shown in experiments 2 and 3, respectively. Note in addition that a doubling or even quadrupling of this value of $\pi$ does not seem to have any significant differential effects on g, c, h, u or v (see Figures 9-13). However, as one would expect, growth is positive and rising, due to the endogenous decrease in the rate of discount $\rho$, and so is the average health level of the population. Note moreover, that income transfers stop after a while. Nonetheless, it is clear that there is very little difference between the outcomes of experiments 3-5, at least from a growth and health benefit perspective. This is not true with respect to costs, however, as shown in Figures 14 and 15.

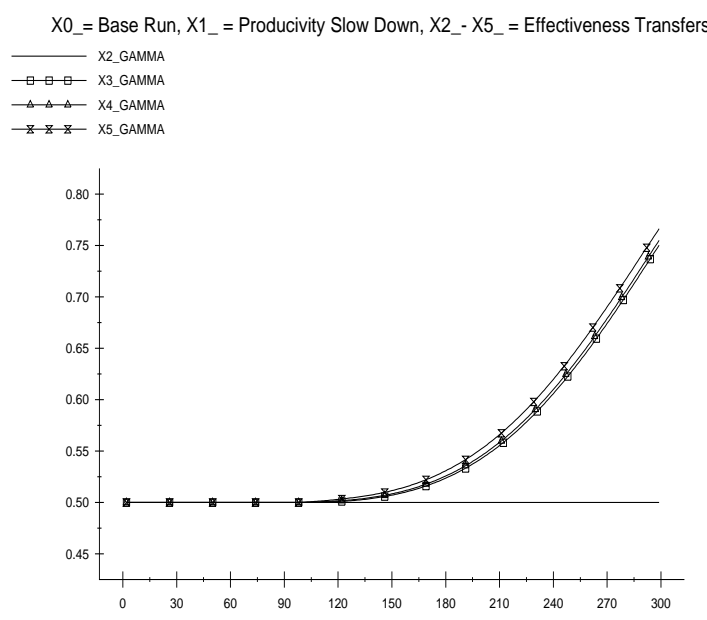

Figure 8

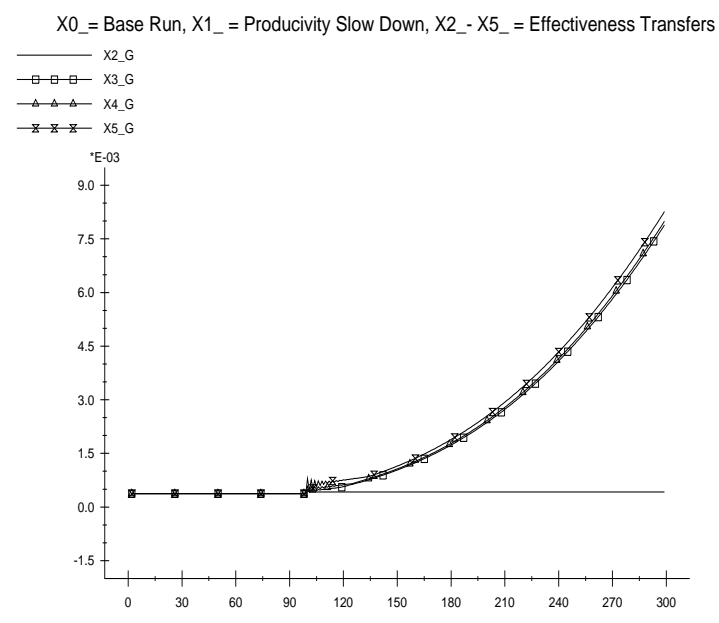

Figure 9 


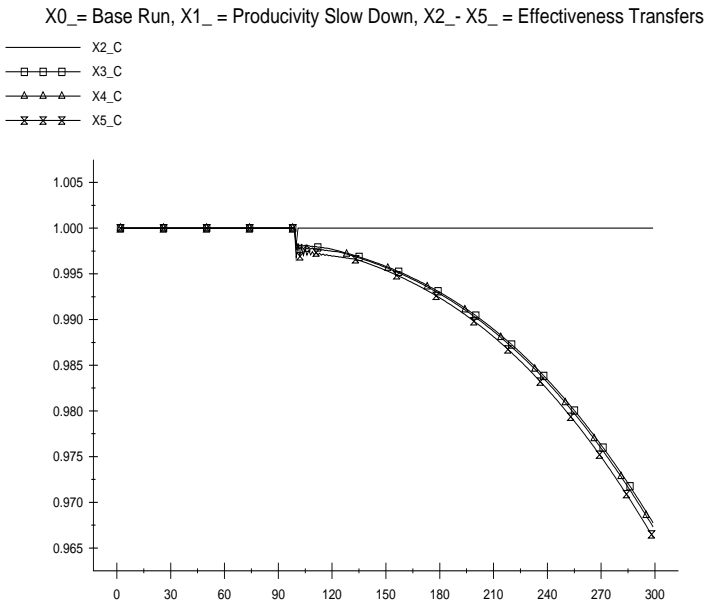

Figure 10

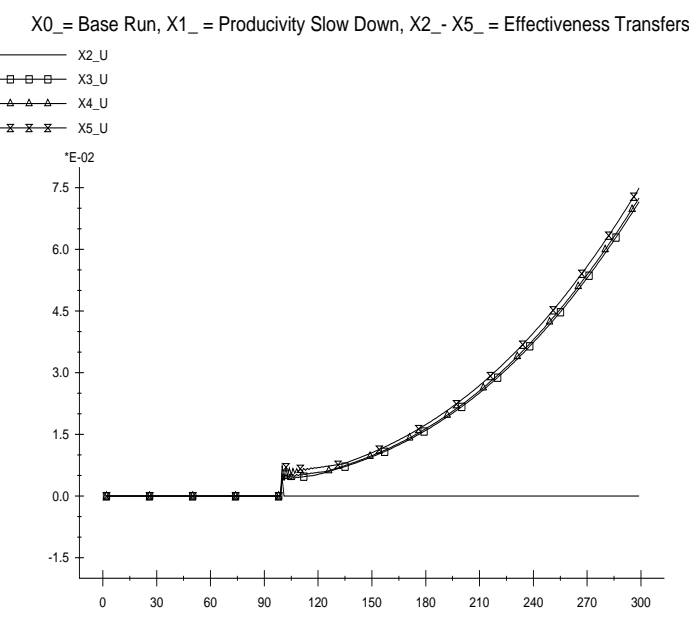

Figure 12

X0_= Base Run, X1_= Producivity Slow Down, X2_- X5_= Effectiveness Transfers X2_TOTAL_TRANSFER

$\square \square \square-X 3 \_$TOTAL_TRANSFER

$\triangle X_{4}$ TOTAL_TRANSFER

$\because \& \&$ X5_TOTAL_TRANSFER

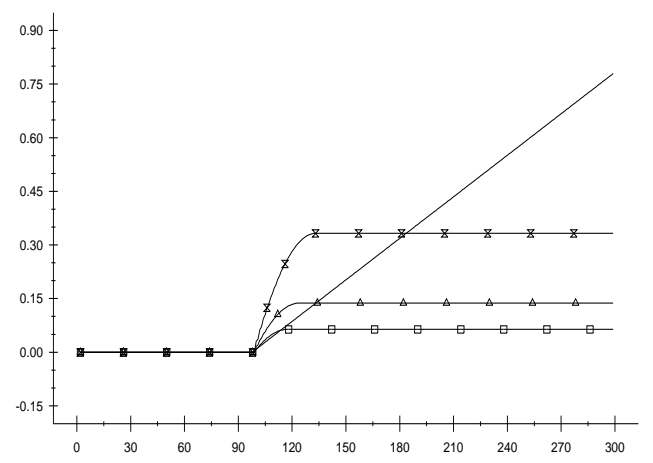

Figure 14

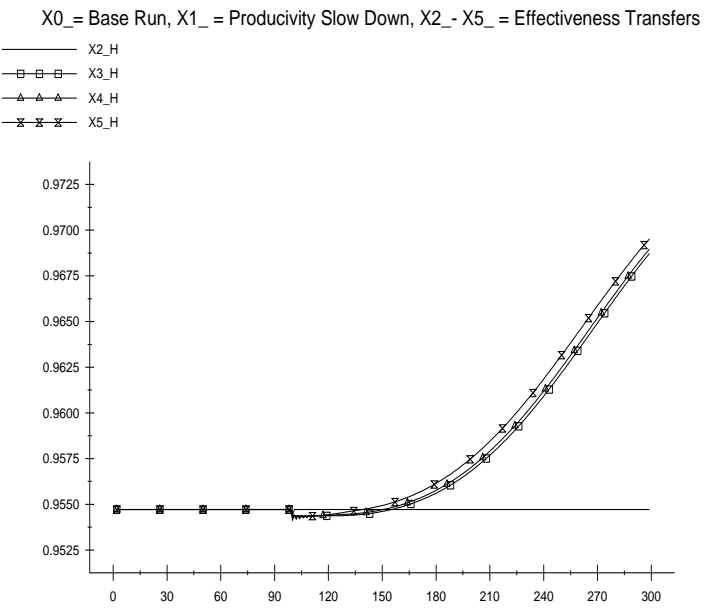

Figure 11

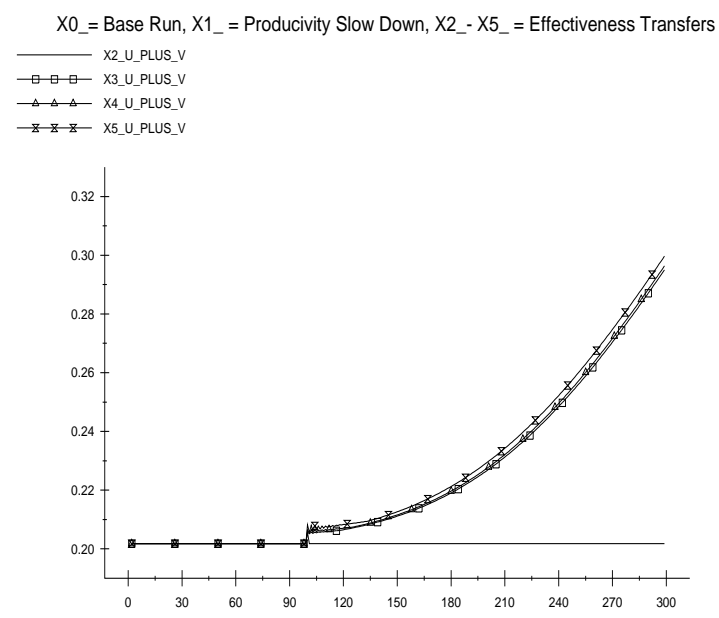

Figure 13

$\mathrm{X} 0_{-}=$Base Run, $\mathrm{X} 1_{-}=$Producivity Slow Down, $\mathrm{X} 2_{-}-\mathrm{X} 5_{-}=$Effectiveness Transfers X2_TRANSFER_Y_RATIO

$\rightarrow \square \square-X_{-}$TRANSFER_Y_RATIO

$\triangle \triangle X_{-}$_TRANSFER_Y_RATIO

$\because \square \square$ X_TRANSFER_Y_RATIO

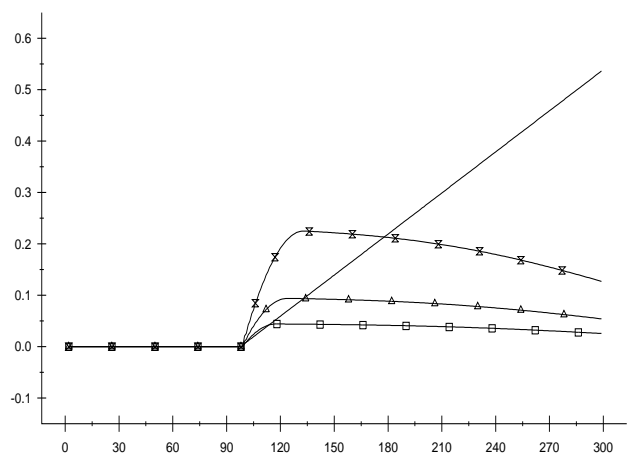

Figure 15 
In Figure 14 we show that after about 20 periods, cumulative transfers level off in experiments $3-5$, because income per head in the receiving country has risen enough to generate the savings (as well as the confidence) necessary to generate and sustain growth. However, we see that in experiment 2 total transfers keep on increasing without bound. This is because in experiment 2 growth never takes off. Moreover, in experiment 2 total transfers as a fraction of the level of income in the receiving country (denoted by TRANSFER_Y_RATIO) keep on rising too, although the transfer-rate is just marginally lower than in experiment 3. In experiments 3-5 this ratio is falling, but from initial levels which rise more than proportionally with the transfer rate.

We conclude then that very low values of $\pi$ may have no beneficial effects at all, as is highlighted in experiment 2 . At the same time the marginal impact of still higher values of $\pi$ on growth performance decreases, while the impact on cumulative transfers increases. However, it is clear that when poor countries can be helped to attain a state where enough savings can be generated to enable the accumulation of physical capital, such a growth process can become self sustaining, given the availability of suitable technologies and external and internal funds. Moreover, as shown in Figures 14-15, if aid is provided in the form of loans, it seems to be in the interest of the receiving country that transfers are just high enough to allow growth to take off, because proportionally higher transfers do not result in proportionally higher growth, although they lead to more than proportionally higher total transfers. In addition to this, the length of the period of adjustment/transition is hardly affected at all. This is underlined by Figures 8-15, which show very similar economic growth and health performance for $\pi$ equal to $0.52,1$ and 2 percent in experiments 3-5, respectively, while the debt/national income ratio in case of experiment 5 is more than twice as large as in experiment 4 and more than 4 times as large as in experiment 3 . However, too little aid in the form of loans, as shown in experiment 2 , would seem to make matters even worse.

\section{Summary and Conclusion}

In this paper we have presented two versions of a simple model of endogenous growth based on the Lucas (1988) model, in which a good health functions as a necessary condition for people to be able to provide labour services. At the same time, health is produced under conditions of decreasing returns, whereas knowledge is produced under conditions of increasing returns. When health does not enter the utility function directly, we find that the health sector has a size which is consistent with maximum economic growth : health is a pure complement to growth from a supply perspective. That is, any re-allocation of labour from the health sector towards the knowledge generating sector would cause a decline in growth.

We also defined a model in which the average health level of the population enters the utility function in a positive way. In order to solve the resulting steady state values of growth and health, we devised a graphical procedure which enables us to state that increases in the demand for health services (either due to an increase in the preference for health, or an increase in the 'health demand base' due to an aging population), will now adversely affect growth : health and growth have become substitutes. This also provides an illustration of Baumols disease with respect to the health sector: a growing part of national income will be spent on health services, thereby negatively affecting the rate of economic growth.

We also concluded that, since the steady state growth rate rises linearly in the average health-level of the population, the productivity of the health-sector is as important a determinant of growth as the productivity of the knowledge generating sector itself. When the health sector is subject to moderate decreasing returns to scale, the impact of changes of the productivity of the health sector on growth may be far larger than for 
equal proportionate changes in the productivity of the knowledge generation process, due to the complementary character of health and economic activity from a supply perspective.

Using the model in which health is an argument of the intertemporal utility function, we have run a number of simulation experiments in order to illustrate the prediction of the model that health will rise and growth will drop when preferences change in the direction of health. We also show that actual growth would lag only little behind steady state growth when the allocation of labour and the propensity to consume would be adjusted in accordance with their steady state values. We showed that the transfer of technology might change a country's state from 'no growth', 'low health' to a state of 'higher health', 'higher growth' by lowering the 'health demand base', and thus freeing resources for growth improving activities, i.e. knowledge generation. Moreover, the transfer of funds to 'no growth', 'low health' countries which do have access to suitable production technologies, can help growth to take off for those countries which suffer from too high values of the rate of discount, if the latter would be endogenously reduced as a result of the growth of income per head. We also found that it is not necessarily the case that a faster rate of transfer of funds leads to significantly better results with respect to growth, both with regard to the rate of growth attained as well as the speed of adjustment to the steady state. Instead, if aid is provided in the form of loans, debts are accumulated faster, while total debts are more than proportionally higher, thus reducing the ability of the receiving country to service its debts. 


\section{Annex A The Lucas Model}

The Hamiltonian is :

$H=e^{-\rho t}\left(\frac{(C / L)^{1-\Theta}-1}{1-\Theta}\right) \cdot L+\lambda\left((A(1-u) L)^{\alpha} K^{1-\alpha}-C\right)+\mu \delta u A$

where $\lambda$ and $\mu$ are the co-state variables. Differentiation of (A.1) w.r.t. the control variables $C$ and $u$, and w.r.t. the state variables $K$ and $A$, result in the following first-order conditions:

$\frac{\partial H}{\partial C}=e^{-\rho t}(C / L)^{-\Theta}-\lambda=0$

$\frac{\partial H}{\partial u}=-\lambda \alpha \frac{Y}{1-u}+\mu \delta A=0$

$\frac{\partial H}{\partial K}=-\frac{d \lambda}{d t}=\lambda(1-\alpha) \frac{Y}{K}$

$\frac{\partial H}{\partial A}=-\frac{d \mu}{d t}=\lambda \alpha \frac{Y}{A}+\mu \delta u$

Substitution of (A.3) into (A.5) gives :

$-\hat{\mu}=\frac{\lambda}{\mu} \frac{\alpha Y}{A}+\delta u=\frac{\delta A(1-u)}{\alpha Y} \cdot \frac{\alpha Y}{A}+\delta u=\delta$

In a situation of steady state growth, $\mathrm{u}$ would have to be constant, which implies that the rate of growth of A would be constant too. Moreover, the rate of growth of the capital stock would have to be equal to the rate of growth of output, in which case the production function itself would lead to the conclusion that :

$\hat{Y}=\alpha \hat{A}+(1-\alpha) \hat{K} \Rightarrow \hat{Y}=\hat{K}=\hat{A}$

Using (A.7) after taking growth rates of (A.3) for constant $\mathrm{u}$, leads to the conclusion that:

$\hat{\lambda}+\hat{Y}-\hat{A}=\hat{\mu} \Rightarrow \hat{\lambda}=\hat{\mu}$

From the macro-economic budget constraint it follows directly that:

$\frac{C}{Y}=1-\hat{K} \frac{K}{Y}$

Hence $\mathrm{C} / \mathrm{Y}$ is constant in the steady state, since $\hat{K}$ is constant, and $\mathrm{K} / \mathrm{Y}$ is constant too. Hence the rate of growth of $C$ equals the rate of growth of $Y$ and hence the rate of growth of A. But (A.2) provides a link between the rate of growth of consumption (and therefore of $A$ ) and $\hat{\lambda}=\hat{\mu}=-\delta$, from which we can obtain the result:

$\hat{A}=\hat{C}=-(\hat{\lambda}+\rho) / \Theta=-(\hat{\mu}+\rho) / \Theta=(\delta-\rho) / \Theta$

where we have used (A.6). (A.10) then implies: 
$u=\hat{A} / \delta=\frac{\delta-\rho}{\delta \Theta}$

Note that (A.9) together with (A.4), (A.6), (A.8) and (A.10), link the optimum saving rate $\mathrm{S}$ with the rate of growth of the system:

$s=\left(1-\frac{C}{Y}\right)=\hat{K} /(Y / K)=-\hat{A} \cdot(1-\alpha) / \hat{\lambda}=\left(\frac{1-\alpha}{\Theta}\right) \cdot\left(1-\frac{\rho}{\delta}\right)$

Equation (A.12) shows that the saving rate rises with the partial output elasticity of capital. It falls with the rate of time preference as reflected by the rate of discount. It is proportional to the intertemporal elasticity of substitution $1 / \Theta$. Furthermore, s rises with the productivity of the knowledge generation process $\delta$. 


\section{Annex B Health as a Complement to Growth}

The Hamiltonian is :

$H=e^{-\rho t}\left(\frac{(C / L)^{1-\Theta}-1}{1-\Theta}\right) \cdot L+\lambda\left((A(1-u-v) h L)^{\alpha} K^{1-\alpha}-C\right)+\mu \delta_{A} u h A$

where $\lambda$ and $\mu$ are the co-state variables. $h$ depends on $v$ through equation (4). Differentiation of (B.1) w.r.t. the control variables C, $u$ and $v$, and w.r.t. the state variables $\mathrm{K}$ and $\mathrm{A}$, results in the following first-order conditions:

$\frac{\partial H}{\partial C}=e^{-\rho t}(C / L)^{-\Theta}-\lambda=0$

$\frac{\partial H}{\partial u}=-\lambda \alpha \frac{Y}{1-u-v}+\mu \delta_{A} h A=0$

$\frac{\partial H}{\partial v}=-\lambda \alpha \frac{Y}{1-u-v}+\lambda \alpha \frac{Y}{h} \frac{\partial h}{\partial v}+\mu \delta_{A} u A \frac{\partial h}{\partial v}=0$

$\frac{\partial H}{\partial K}=-\frac{d \lambda}{d t}=\lambda(1-\alpha) \frac{Y}{K}$

$\frac{\partial H}{\partial A}=-\frac{d \mu}{d t}=\lambda \alpha \frac{Y}{A}+\mu \delta_{A} u h$

Along with the accumulation constraint :

$\frac{d A}{d t}=\delta_{A} u h A$

Substitution of (B.3) into (B.6) gives :

$-\hat{\mu}=\frac{\lambda}{\mu} \frac{\alpha Y}{A}+\delta_{A} u h=\frac{\delta_{A} A(1-u-v)}{\alpha Y} \cdot \frac{\alpha Y}{A}+\delta_{A} u=\delta_{A} h(1-v)$

Substitution of (B.3) into (B.4) results in :

$(\lambda \alpha Y) \cdot\left(\frac{-1}{1-u-v}+\frac{z_{1}}{v}+\frac{u \cdot z_{1}}{v \cdot(1-u-v)}\right)=0$

which leads to the conclusion that:

$v=\frac{z_{1}}{1+z_{1}}=\beta$

Hence, $\mathrm{v}$ has a very simple solution. It follows that an unproductive health system, as reflected by a low value of $\beta$, should be relatively small. The corresponding steady state health-level $\bar{h}$ can directly be obtained from (4) by substituting (B.10):

$\bar{h}=\left(\beta^{\beta} \cdot \frac{\delta_{h}}{\zeta}\right)^{1 /(1-\beta)}=z_{0} v^{z_{1}}$ 
In a situation of steady state growth, $u$ would have to be constant, which implies that the rate of growth of A would be constant too. Moreover, the rate of growth of the capital stock would have to be equal to the rate of growth of output, in which case the production function itself would lead to the conclusion that :

$\hat{Y}=\alpha \hat{A}+(1-\alpha) \hat{K} \quad \Rightarrow \quad \hat{Y}=\hat{K}=\hat{A}$

Taking growth rates, while assuming that in the steady state the health level is constant, allows us to rewrite (B.3) and (B.4) as :

$\hat{\lambda}+\hat{Y}=\hat{\mu}+\hat{A}$

(B.12) and (B.13) taken together imply :

$\hat{\mu}=\hat{\lambda}$

But substitution of (B.3) into (B.6) gives:

$-\hat{\mu}=\delta_{A} \cdot(1-v) \bar{h}=\delta_{A} \cdot(1-\beta) \bar{h}$

where we have used (B.10). In the steady state (which implies that C/Y is constant), (B.2), (B.14) and (B.15) imply that :

$g=\hat{A}=\hat{C}=\hat{Y}=\hat{K}=\frac{\delta_{A}(1-v) h(v)-\rho}{\Theta}=\frac{\delta_{A}(1-\beta) \bar{h}-\rho}{\Theta}$

(B.16) can directly be compared with (A.10), since $(1-\beta) \bar{h}$ in (B.16) is the amount of labour (measured in health units) not absorbed by the health sector, and which is therefore available for alternative uses.

Note that the rate of growth of the economy now depends on the productivity parameters of the health sector, through the 'term' $(1-\beta) h$. Note moreover from partial differentiation of (B.16) with respect to $\mathrm{v}$, that $\mathrm{g}$ is maximised when $v=\beta$. 


\section{Annex C Health as a Substitute of Growth}

The Hamiltonian is :

$H=e^{-\rho t}\left(\frac{\left\{(h / \bar{h})^{\gamma}(C / L)\right\}^{1-\Theta}-1}{1-\Theta}\right) \cdot L+\lambda\left((A(1-u-v) h L)^{\alpha} K^{1-\alpha}-C\right)+\mu \delta_{A} u h A$

In comparison with our first model, only the first order conditions regarding $\mathrm{C}$ and $\mathrm{v}$ are affected. The other first order conditions are the same as in Annex B. Hence (B.2) will now read:

$\frac{\partial H}{\partial C}=e^{-\rho t}(h / \bar{h})^{\gamma \cdot(1-\Theta)} \cdot(C / L)^{-\Theta}-\lambda=0$

(C.2) still provides the same constraint on the growth rate of $\mathrm{C}$ and the co-state variable $\lambda$. In that sense the model is not changed. This also holds true w.r.t. the first order condition for $\mathrm{u}$ :

$\frac{\partial H}{\partial u}=-\lambda \alpha \frac{Y}{1-u-v}+\mu \delta_{A} h A=0$

However, (B.4) now becomes:

$\frac{\partial H}{\partial v}=\gamma e^{-\rho t}\left\{(h / \bar{h})^{\gamma}(C / L)\right\}^{1-\Theta} \cdot \frac{z_{1}}{v} \cdot L-\lambda \alpha \frac{Y}{1-u-v}+\lambda \alpha Y \frac{z_{1}}{v}+\mu \delta_{A} u h A \frac{z_{1}}{v}=0$

Assuming a constant steady state saving rate s, C can be written as $C=(1-s) Y$. Substitution of $\mathrm{C}=(1-\mathrm{s}) \mathrm{Y}$ as well as (C.2) and (C.3) into (C.4) then results in:

$\frac{(1-s) \gamma z_{1}}{v}+\frac{\alpha z_{1}}{v}-\frac{\alpha}{1-u-v}+\frac{\alpha u z_{1}}{(1-u-v) v}=0$

where we have made use of the relation $\frac{\partial h}{\partial v}=z_{1} h / v$.

Substitution of (C.3) into the first order constraint regarding the state variable A, leads to the conclusion that:

$-\hat{\mu}=\delta_{A} h(1-v)$

which, together with the conditions that $\hat{Y}=\hat{K}=\hat{A}=\hat{C}=-(\hat{\mu}+\rho) / \Theta$ (these conditions follow directly from the steady state assumption in combination with (C.3) and the production function), imply that:

$g=\hat{A}=\hat{Y}=\hat{C}=\left(\delta_{A} h(1-v)-\rho\right) / \Theta$

But from the knowledge generation equation we have :

$g=\hat{A}=\delta_{A} u h$

Moreover, it follows from (C.2) and $-\hat{\lambda}=(1-\alpha) Y / K$ in combination with $s=\hat{K} /(Y / K)$ that :

$s=(1-c)=(1-\alpha) g /(\Theta g+\rho)$ 
Substitution of (C.8) into the nominator of (C.9) and (C.7) into the denominator of (C.9) then directly results in:

$(1-c)=(1-\alpha) u /(1-v) \Rightarrow u=(1-c)(1-v) /(1-\alpha)$

Substitution of $c=1-\mathrm{s}$ as well as (C.10) into (C.5) and solving for v results in:

$v=\frac{c(c-\alpha)+\alpha(1-\alpha) / \gamma}{c(c-\alpha)+\alpha(1-\alpha) /(\gamma \beta)}$

Note that for $c=\alpha, v=\beta$, while for c going to infinity v would asymptotically approach a value of 1 . However $c=1$ is the maximum value of $c$, in which case $v=\beta \cdot(\alpha+\gamma) /(\alpha+\gamma \beta)>\beta$.

Defining $f=c(c-\alpha)$, equations (10.A)-(10.D) follow directly from (C.11), (C.9) and (C.7), whereas equation (10.E) is the same as (C.10). 


\section{Annex D Mappings}

Note that the simultaneous sub-system described by (10.A)-(10.D) can be represented as a 'four quadrant diagram', because each of the dependent variables depends on only one other variable, which is different for each of the dependent variables. So, given an initial value of $c$, $f$ can be calculated, then $v$, then $g$, and then $c$ again. Hence, this particular 'mapping process' maps a point of the c-axis onto the c-axis again by 'going round' in a counter clock-wise direction in the four quadrant diagram depicted in Figure D.1. We may describe this mapping process by $\mathrm{O}^{\mathrm{n}}=\mathrm{M}^{\mathrm{n}}(\mathrm{X})$, where $\mathrm{O}^{\mathrm{n}}$ denotes the outcome of the mapping process $\mathrm{M}()$ after $n$ 'mapping rounds' starting from an initial point $X$ in the range B-C in Figure D.1 below, which combines Figures 1 and 2. It is a special combination, however, for the extremum of $g=g(v)$ has a value which is exactly equal to $\rho /(1-\Theta)$, while the largest root of $g(v)=0$ coincides with $v^{*}=v(f(c(0)))$. We use Figure D.1 to illustrate that the equilibrium solution of (10.A-10.D) can be obtained by means of an iterative procedure which uses a continuous mapping of $g$ onto itself.

There are now three important questions to be answered. The first question is whether there exists points $c^{*}$ such that $c^{*}=M^{n}\left(c^{*}\right)$, for all $n$. The second question is whether $c^{*}$ would be unique if it exists. The third question is whether any $X$ would lead to $c^{*}$ if $c^{*}$ exists, i.e. whether $c^{*}=M^{n}(X)$ for large enough $n$ and for any $X$. If there is such $a c^{*}$, then, obviously, the first order conditions of the Hamiltonian system have a unique solution $\left(\mathrm{c}^{*}, \mathrm{f}^{*}, \mathrm{v}^{*}, \mathrm{~g}^{*}\right)$. Moreover, a numerical solution could easily be obtained by directly applying the mapping procedure. This is of considerable practical importance, since even a system as simple as (10.A-10.D) can not readily be solved in an analytical fashion.

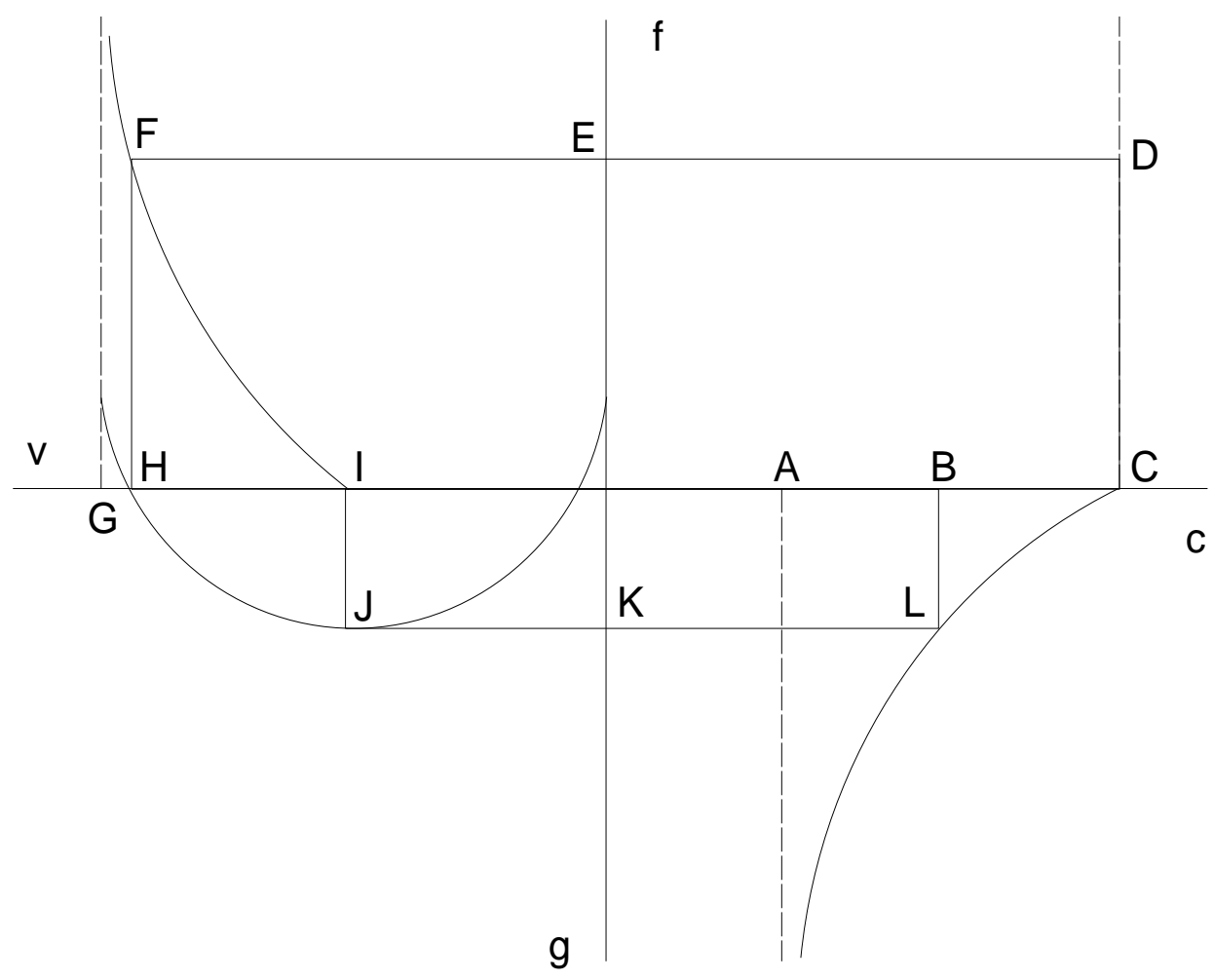

Figure D.1 
In order to be able to address the questions raised above, it is useful to define the concept of a 'line-segment pair'. We use it to indicate the combination of 'top' and 'bottom' line-segments which can be obtained by mapping a certain value $c^{\prime}$ from the range B-C on $\mathrm{f}$ and $\mathrm{v}$, which gives the associated 'top' horizontal line-segment, and then on $\mathrm{g}$ and c, which gives the corresponding 'bottom' horizontal line-segment. (Point $C$ in Figure D.1, for example, generates the 'top' line-segment F-D, and the 'bottom' line-segment $\mathrm{H}-\mathrm{C}$.) Let us denote the 'top' line-segment associated with point $\mathrm{c}^{\prime}$ in the range B-C in the n-th 'mapping round' by $\mathrm{T}^{\mathrm{n}}\left(\mathrm{c}^{\prime}\right)$, and the corresponding 'bottom' line-segment by $\mathrm{B}_{\mathrm{n}}\left(\mathrm{c}^{\prime}\right)$, respectively. Let us furthermore denote the length of a line-segment $S$ by $L(S)$. Note furthermore that for any interior point $c^{*}$ such that $L\left(B^{n}\left(c^{*}\right)\right)=L\left(T^{n}\left(c^{*}\right)\right)$, it necessarily follows that $c^{*}$ solves (10.A)-(10.D), because the left-hand bounds of $B^{n}\left(c^{*}\right)$ and $\mathrm{T}^{\mathrm{n}}\left(\mathrm{c}^{*}\right)$ have the same horizontal co-ordinates by construction. But if $\mathrm{L}\left(\mathrm{B}^{\mathrm{n}}\left(\mathrm{c}^{*}\right)\right)=\mathrm{L}\left(\mathrm{T}^{\mathrm{n}}\left(\mathrm{c}^{*}\right)\right)$, then the right-hand bounds of $\mathrm{B}^{\mathrm{n}}\left(\mathrm{c}^{*}\right)$ and $\mathrm{T}^{\mathrm{n}}\left(\mathrm{c}^{*}\right)$ also have the same horizontal co-ordinates. Hence the mapping process $M$ has resulted in a fixed point $\mathrm{c}^{*}$ (and therefore in associated fixed points $\mathrm{f}^{*}, \mathrm{v}^{*}$ and $\left.\mathrm{g}^{*}\right)$. Hence $\mathrm{c}^{*}=\mathrm{M}^{\mathrm{n}}\left(\mathrm{c}^{\prime}\right)$, and $\mathrm{c}^{*}=\mathrm{M}^{\mathrm{k}}\left(\mathrm{c}^{*}\right)$, for all $k>=0$.

Figure D.1 provides direct clues with regard to constraints on the parameters of the system which would make a mapping process $\mathrm{M}()$ as described above possible at all. For, Figure D.1 indicates that sufficient conditions for c to be an interior point of B-C, are that the length of line-segment I-J is smaller than the length of line-segment B-L, while at the same time $\mathrm{g}(\mathrm{v}(\mathrm{f}(1)))>0$, i.e. the largest root of $\mathrm{g}(\mathrm{v})$ should be to the left of point $H$. In order to see this, note that $C=M^{n}(C)$ for $n>=0$, i.e. $C$ is a fixed point of the Figure D. 1 as it is. When we would require $\left(\mathrm{g}(\mathrm{v}(\mathrm{f}(\mathrm{C})))>0\right.$ and $\mathrm{L}(\mathrm{I}-\mathrm{J})>\mathrm{L}(\mathrm{B}-\mathrm{L})$, then $\mathrm{M}^{1}(\mathrm{C})$ would map the original point $\mathrm{F}$ onto a point $\mathrm{H}^{\prime}$ which is directly below the original point $\mathrm{H}$ on the $\mathrm{g}(\mathrm{v})$ curve, and a point on the $\mathrm{c}(\mathrm{g})$ curve which in turn maps onto an interior point of $\mathrm{B}-\mathrm{C}$. The important thing to notice is that starting from point $\mathrm{C}$ we would have $L\left(T_{n}(C)\right)>=L\left(B_{n}(C)\right)$ for all $n>=0$. However, both $L\left(T_{n}(C)\right)$ and $L\left(B_{n}(C)\right)$ fall in a monotonous fashion with $n .{ }^{34}$ But the smallest lengths to which $\mathrm{L}\left(\mathrm{T}^{\mathrm{n}}(\mathrm{C})\right)$ and $L\left(B^{n}(C)\right)$ could fall are $L(I-B)$ and $L(J-B)$, respectively, where $L(J-B)>L(I-B)$ under the conditions described above. But we also know that as long as $\left.\mathrm{L}^{\mathrm{n}}(\mathrm{T})\right)>\mathrm{L}\left(\mathrm{B}^{\mathrm{n}}(\mathrm{C})\right)$, the mapping process $\mathrm{M}()$ will bring the line-segments $\mathrm{T}^{\mathrm{n}}(\mathrm{C})$ and $\mathrm{B}^{\mathrm{n}}(\mathrm{C})$, nearer to their lower limits. Since the lower limit of the 'bottom' line-segment exceeds the lower-limit of the 'top' line-segment, there must be a $k>0$ where $L\left(T^{k}(C)\right)=L\left(B^{k}(C)\right)$, i.e. $c^{*}=M^{k}(C)$ solves the first order conditions of the Hamiltonian system.

Now consider point B in Figure D.1. In the Figure as it is, B is a fixed point. When we would require $g(v(f(1)))>0$ and $\mathrm{L}(\mathrm{I}-\mathrm{J})<\mathrm{L}(\mathrm{B}-\mathrm{L})$, B would have a tendency to move closer to point $C$. Both the line-segments I-B and J-L would expand in the process. But I-B could expand to a maximum length $\mathrm{L}(\mathrm{F}-\mathrm{D})$, whereas $\mathrm{J}-\mathrm{L}$ could expand to a maximum length $L\left(G^{\prime}-C\right)$, where $G^{\prime}$ is in between $G$ and $H$ in order to ensure $g(v(f(1)))>0$. Obviously, $L\left(G^{\prime}-C\right)>L(F-D)$. Note that starting from $B$, we would necessarily have $\mathrm{L}\left(\mathrm{T}^{\mathrm{n}+1}(\mathrm{~B})\right)>=\mathrm{L}\left(\mathrm{B}^{\mathrm{n}}(\mathrm{B})\right)$, and both $\mathrm{L}\left(\mathrm{T}^{\mathrm{n}+1}(\mathrm{~B})\right)$ and $\mathrm{L}\left(\mathrm{B}^{\mathrm{n}}(\mathrm{B})\right)$ are monotonically increasing with $n$ as long as $L\left(T^{n+1}(B)\right)>L\left(B^{n}(B)\right)$. Hence $L\left(T^{n+1}(B)\right)$ would tend to converge to a limit which is smaller than the limit of $L\left(B^{n}(B)\right)$. Therefore, there must be a $k>=0$ for which $c^{* *}=M^{k+1}(B)=M^{k}(B)$ is a fixed point for $c$. If $c^{* *}>c^{*}$, then we would have the mapping process $M()$ converging to $c^{*}$ again. So, by virtue of the fact that $c^{*}=M^{k}(C)$, we conclude that if $B$ has its own fixed point, then it should be an interior point of the range $B-\mathrm{C}^{*}$.

34 This follows from the fact that a downward movement of the line-segment F-D makes F move to the right and $\mathrm{D}$ to the left. Hence $\mathrm{L}(\mathrm{F}-\mathrm{D})$ decreases monotonically when the line-segment F-D moves down. This also holds for $\mathrm{L}(\mathrm{H}-\mathrm{C})$ when the line-segment $\mathrm{H}-\mathrm{C}$ moves down. 


\section{Annex E Parameter Restrictions}

The possibility that there are a number of solutions to the Hamiltonian system instead of one, is highlighted in Figure 1, where the mapping $\mathrm{z}(\mathrm{g})=\mathrm{v}(\mathrm{f}(\mathrm{c}(\mathrm{g})))$ defines a negatively sloped curve in the $(\mathrm{v}, \mathrm{g})$ plane. That the slope is negative, can easily be verified, because $\frac{\partial z}{\partial g}=\frac{\partial v}{\partial f} \cdot \frac{\partial f}{\partial c} \cdot \frac{\partial c}{\partial g}$, where only c depends negatively on $g$, and the partial derivatives of $v$ with respect to $\mathrm{f}$, and $\mathrm{f}$ with respect to $\mathrm{c}$ are positive for $\alpha \leq c \leq 1$. The lowest value of $\mathrm{v}$ is obtained for $g=\rho /(1-\Theta)$, while the highest value for $v$, i.e. $v^{*}=\beta \cdot(\gamma+\alpha) /(\gamma \cdot \beta+\alpha)$ is obtained for $g=0$. When $v=z(g)$ would be convex in $g$, and when the growth rate $g$ as a function of $v$, i.e. $g=g(v)$, would be such that its maximum value is smaller than $\rho /(1-\Theta)$, while $g\left(v^{*}\right)>0$, then $v=z(g(v))$ defines a unique $v$. In general, however, $z()$ could also be concave in $\mathrm{g}$, which opens up the possibility of multiple roots of $\mathrm{v}=\mathrm{z}(\mathrm{g}(\mathrm{v}))$. This follows directly from the fact that:

$\frac{\partial^{2} v}{\partial g^{2}}=\frac{\partial^{2} v}{\partial f^{2}} \cdot\left\{\frac{\partial f}{\partial c} \cdot \frac{\partial c}{\partial g}\right\}^{2}+\frac{\partial^{2} f}{\partial c^{2}} \cdot \frac{\partial v}{\partial f} \cdot\left\{\frac{\partial c}{\partial g}\right\}^{2}+\frac{\partial^{2} c}{\partial g^{2}} \cdot \frac{\partial v}{\partial f} \cdot \frac{\partial f}{\partial c}$

where the first term on the right hand side of (E.1) is negative because of the concavity of $\mathrm{v}(\mathrm{f})$ in $\mathrm{f}$, and the remaining terms are all positive. Unfortunately, it is not easy to obtain the roots for (E.1) in an analytical fashion and then determine whether or not $\mathrm{v}=\mathrm{z}(\mathrm{g})$ would be concave and/or convex for sub-ranges of $\alpha \leq c \leq 1$. However, as a second best option we have evaluated (E.1) in the points $g=0$ and $g=\rho /(1-\Theta)$. We also evaluated the first order partial derivatives in those same points. In order for $\mathrm{v}=\mathrm{z}(\mathrm{g})$ to be convex in $g$ it is then necessary that the first partial derivative rises for increasing $\mathrm{g}$, whereas both second order partial derivatives would have to be positive. Hence, we would have:

\begin{tabular}{|l|l|}
\hline$\frac{\partial v}{\partial g} I_{g=0}$ & $-\frac{\alpha \beta \gamma(1-\beta)(2-\alpha)}{\rho(\alpha+\beta \gamma)^{2}}$ \\
\hline$\frac{\partial v}{\partial g} I_{g=\rho /(1-\Theta)}$ & $-\frac{\beta \gamma(1-\Theta)^{2}(1-\beta)}{\rho}$ \\
\hline$\left.\frac{\partial^{2} v}{\partial g^{2}}\right|_{g=0}$ & $-\frac{2 \alpha \beta \gamma(1-\beta)\left\{\alpha^{2}(\beta \gamma+\Theta+1)+\alpha(\beta \gamma(\Theta-3)-2 \Theta-1)+\beta \gamma(3-2 \Theta)\right\}}{\rho^{2}(\alpha+\beta \gamma)^{3}}$ \\
\hline$\left.\frac{\partial^{2} v}{\partial g^{2}}\right|_{g=\rho /(1-\Theta)}$ & $\frac{2 \beta \gamma(1-\Theta)^{3}(1-\beta)(\alpha(\beta \gamma(\Theta-1)+2 \Theta-1)-\Theta+1)}{\alpha \rho^{2}}$ \\
\hline
\end{tabular}

Table E.1

It is now easy to show that in order for $\mathrm{v}=\mathrm{z}(\mathrm{g})$ to be convex, we must have:

$\left.\frac{\partial v}{\partial g}\right|_{g=\rho /(1-\Theta)} \geq\left.\frac{\partial v}{\partial g}\right|_{g=0} \Rightarrow\left\{\left.\frac{\partial v}{\partial g}\right|_{g=\rho /(1-\Theta)}\right\}\left\{\left.\frac{\partial v}{\partial g}\right|_{g=0}\right\} \leq 1 \Rightarrow \gamma \leq \frac{\alpha}{\beta(1-\theta)}\left\{\sqrt{\frac{2-\alpha}{\alpha}}-1\right\}$ 
Obviously, the inequalities in (E.2) would have to be reversed to ensure concavity of $\mathrm{v}=\mathrm{z}(\mathrm{g})$ in $\mathrm{g}$. Apart from (E.2), the fact that the second order partial derivatives need to be positive for convexity of $\mathrm{v}=\mathrm{z}(\mathrm{g})$ implies that :

$\left.\frac{\partial^{2} v}{\partial g^{2}}\right|_{g=0} \geq 0 \Rightarrow \gamma \leq \frac{\alpha(1+2 \Theta)-(1+\Theta) \alpha^{2}}{\beta\left(\alpha^{2}-\alpha(3-\Theta)+3-2 \Theta\right)}$

The nominator of (E.3) has roots $\alpha=0$ and $\alpha=(1+2 \Theta) /(1+\Theta) \geq 1$. Hence the nominator is positive for all relevant values of $\alpha$. The denominator is also positive, since it has no real roots :

$\alpha_{1,2}=\{3-\Theta \pm \sqrt{(\Theta+3)(\Theta-1)}\} / 2$

where $(\Theta+3)(\Theta-1) \leq 0$ for $0<\Theta \leq 1$. Hence the denominator has no real roots, or, for $\Theta=1, \alpha=1$ is the only real root. But in that case the requirement that $0<\alpha \leq 1$ would ensure that the denominator is non negative.

Finally, we have :

$\left.\frac{\partial^{2} v}{\partial g^{2}}\right|_{g=\rho /(1-\Theta)} \geq 0 \Rightarrow \gamma \leq \frac{1}{\beta}\left\{\frac{1-\alpha}{\alpha}+\frac{\Theta}{1-\Theta}\right\}$

In order to see what range of variation these constraints leave for $\gamma$, it is useful to calculate some numbers based on the assumption that $\alpha=0.65, \Theta=0.5$. For these particular values we find that (E.2) is reduced to $\gamma \leq 1.35 / \beta$, (E.3) is reduced to $\gamma \leq 0.84 / \beta$, while (E.5) is reduced to $\gamma \leq 1.54 / \beta$. Hence, for a size of the health-sector of the order of 0.1 , i.e. $\beta$ is of the order of 0.1 , we would find that the maximum value of $\gamma$ is of the order of 10 . Hence, for 'reasonable' values of the parameters, the restrictions on $\gamma$ do not seem to be very strong.

Apart from the restrictions which follow from the assumed convexity of $\mathrm{v}=\mathrm{z}(\mathrm{g})$, there are some additional parameter restrictions on the health-parameters due to the fact that we have required :

$g(v=\beta) \leq \rho /(1-\Theta)$

$g(v=\beta(\alpha+\gamma) /(\beta \gamma+\alpha)) \geq 0$

For the purpose of the paper we simply assume that these restrictions are fulfilled. As a check on the working of the model we have generated random parameter sets in accordance with all the restrictions mentioned above, and we have established that starting from either $g=0$ or $g=\rho /(1-\Theta)$ using the mapping process implied by (10.A-10.D), does indeed lead to a convergence of the solution of the Hamiltonian to the unique solution implied by the convexity of $\mathrm{v}=\mathrm{z}(\mathrm{g})$ and the restrictions on $\mathrm{g}(\mathrm{v})$ as given by (E.6) and (E.7). 


\section{Annex F Parameter Changes and System Responses}

It should be noted here that the 'health-parameters' $\beta, \delta_{h}, \zeta, \gamma$ only influence the shape of the curves $g=g(v)$ and $v=v(f)$. The 'non-health parameters' $\Theta, \rho, \delta_{A}$ influence the curves $\mathrm{c}=\mathrm{c}(\mathrm{g})$ and $\mathrm{g}=\mathrm{g}(\mathrm{v})$.

\section{Changes in Non-Health Parameters}

Consider a rise in the value of $\Theta$, which corresponds to a fall in the intertemporal elasticity of substitution. This would leave the points of intersection of $\mathrm{g}(\mathrm{v})$ with the horizontal axis unchanged, whereas it would decrease the value of $g$ for any given value of $\mathrm{v}$. It would also lead to a counter-clock wise rotation of the curve $\mathrm{c}=\mathrm{c}(\mathrm{g})$ in Figure 1. The latter would stretch the curve $\mathrm{H}-\mathrm{H}^{\prime}$ in a downward direction, which in turn would lead to a new solution of the Hamiltonian system with lower growth and higher health. This change is re-enforced by the fact that the positive part of $\mathrm{g}(\mathrm{v})$ itself, i.e. the part below the horizontal v-axis, moves in an upward direction. This leads to the 'final' solution $X^{\prime}$ which has even lower growth and higher health. In addition to this, the propensity to consume (i.e. the complement of the propensity to save/invest) has risen, which is in line with the fact that a rise in $\Theta$ indicates a decrease in the willingness of people to substitute current consumption for future consumption. Both changes in the curves $\mathrm{v}=\mathrm{z}(\mathrm{g})$ and $\mathrm{g}=\mathrm{g}(\mathrm{v})$ are depicted in Figure F.1, where we only represent the fourth quadrant associated with the $\mathrm{g}, \mathrm{v}-\mathrm{plane}$ in the same orientation as in Figures 1 and 2.

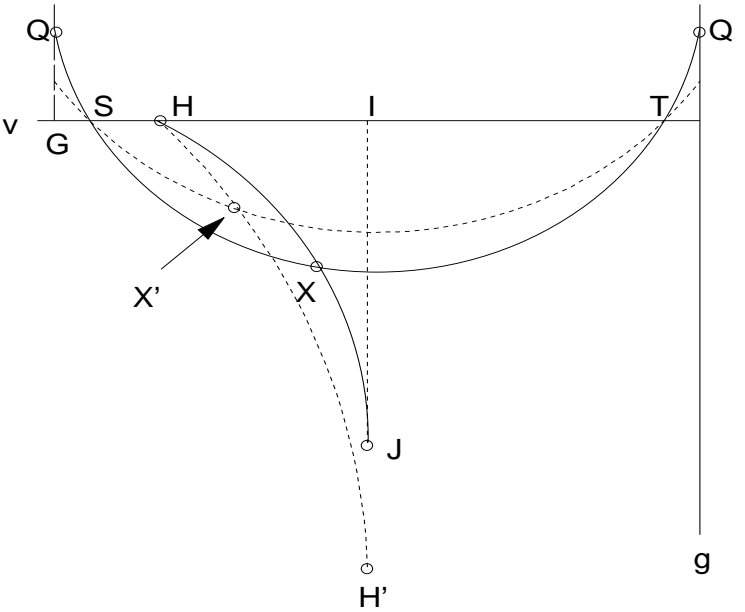

Figure F.1 A rise in $\Theta$

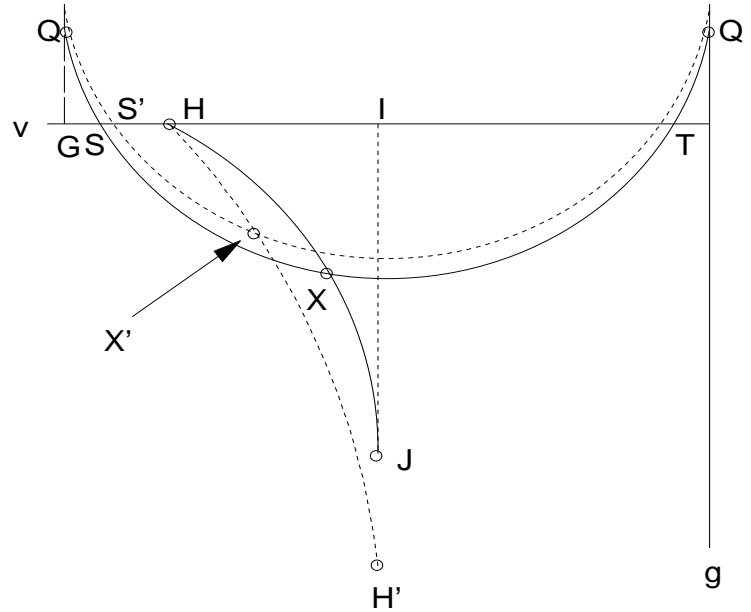

Figure F.2 A rise in $\rho$

Now consider a rise in the rate of discount $\rho$. This would have two effects. First the curve $g=g(v)$ in Figure 2 would shift in an upward direction without changing its curvature. Secondly, the curve $c=c(g)$ in Figure 1 would rotate in a counter-clockwise direction, for c would be larger for any value of $\mathrm{g}$. Consequently, the equilibrium values of both $\mathrm{c}$ and $\mathrm{v}$ rise, while the equilibrium value of $\mathrm{g}$ falls. $\mathrm{h}$ rises and $\mathrm{u}$ falls while $\mathrm{c}$ rises, which seems logical, given the preferences of people to have their consumption and health returns sooner rather than later. Note, however, that the upward shift of $\mathrm{g}=\mathrm{g}(\mathrm{v})$ brings the roots of $\mathrm{g}(\mathrm{v})=0$ closer together. If the largest root would fall below $\mathrm{V}^{*}$, then there are two admissible solutions to the Hamiltonian system, i.e. a low-growth, high-health solution (i.e. the one with $\mathrm{v}$ closest to $\mathrm{v}^{*}$ ), and a high-growth, low-health solution. We come back to this later. 
Now consider a rise in the productivity of the knowledge sector, i.e. $\delta_{A}$. This would only affect the position and shape of the curve $g=g(v) . g(v)$ would shift in a downward direction, while the points of intersection of $g(v)$ with the vertical axes at $v=0,1$ would be unaffected. This would result in a new equilibrium in which we would have lower $c$, higher growth and lower health. The latter may seem to be somewhat puzzling perhaps, but it can be understood once one realises that an increase in $\delta_{A}$ would also increase the opportunity cost of maintaining the original size of the health-sector. The results are depicted in Figure F.3.

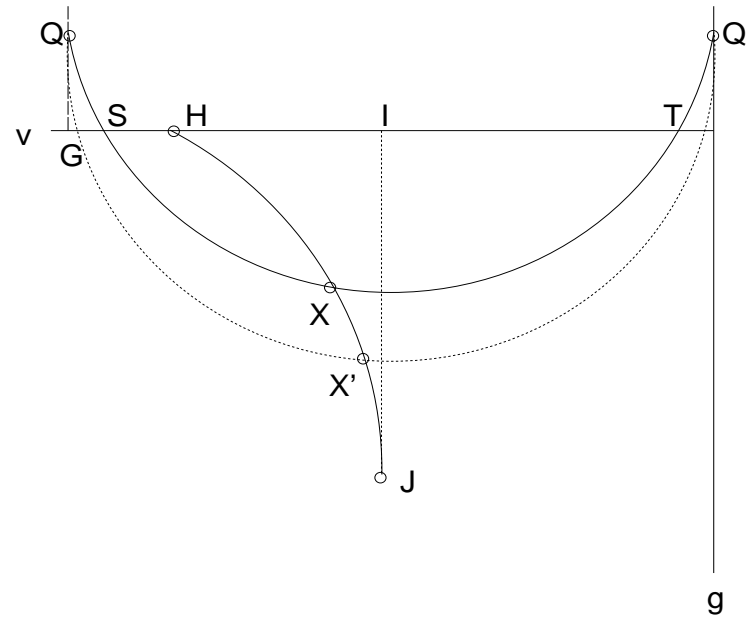

Figure F.3 A rise in $\delta_{A}$

\section{Changes in Health Parameters}

Now consider a rise in $z_{0}$, which might be caused either by an increase in the productivity of the health sector (i.e. $\delta_{h}$ ), or a decrease in the rate of decay of health (i.e. $\zeta$ ), or a decrease in the size of the population. If either of these things would happen, then this would stretch the curve $\mathrm{g}(\mathrm{v})$ in a downward direction without changing its points of intersection with the vertical axes for $v=0,1$. Neither would the horizontal location of the extremum of $g=g(v)$ change. This being the case, we see that the solution changes to a higher growth, smaller health-sector (i.e. smaller v) solution. The fact that $v$ falls, does however not necessarily imply that health itself falls, since the fall in health induced by a fall in $\mathrm{v}$, is more than compensated by the rise in the productivity of the health sector. This can be understood by considering that an increase in $z_{0}$ would 'free' scarce resources in the health sector without an accompanying reduction in the average health level of the population. These 'additional' resources should then be allocated over their alternative uses (including the generation of health), which would involve a rise in the average health level. The shift in the $g=g(v)$ curve implies that the educational sector as well as growth itself would expand, whereas saving rates would have to increase in order to be able to sustain the larger growth rate. Note that because of the way in which $z_{0}$ enters the $g=g(v)$ function, the results are directly comparable to the results obtained for a change in $\delta_{A}$, which were depicted in Figure F.3. Hence, we do not repeat that figure here.

Now consider a rise in the valuation of a good health, i.e. an increase in $\gamma$. In this case the curve $v=v(f)$ in Figure 1 would rotate in a counter-clockwise direction. This has the effect of moving the point of intersection of $\mathrm{v}=\mathrm{z}(\mathrm{g})$ with the $\mathrm{v}$-axis to the left, whereas $v=z(\rho /(1-\Theta))$ would remain unchanged. This would raise $\mathrm{v}$, lower $\mathrm{g}$, and raise $\mathrm{c}$, and hence lower $u$ (by virtue of equation (10.E)). Hence, we would have higher average 
health-levels and lower growth levels. Note that, like a change in the parameters which define the shape of $\mathrm{g}(\mathrm{v})$, a higher preference for a good health can cause the occurrence of multiple solutions, even when $\mathrm{v}=\mathrm{z}(\mathrm{g})$ would be convex. The question is then which of the two solutions would be the relevant one. The answer to this question can be found by considering what would happen to those two solutions when $\gamma$ would go on increasing after a second point of intersection between $g=g(v)$ and $v=z(g)$ appears in point $S$ in Figure F.4, and which starts moving in the direction of the first point of intersection for ever increasing values of $\gamma$. Obviously, the first point of intersection between $g=g(v)$ and $v=z(g)$, i.e. the high-growth, low-health equilibrium, still describes behaviour which is logical from an economic point of view, while the high-health, low-growth solution involves a contraction of the health-sector when people would increase their preference for a good health. The latter result is counter intuitive, to say the least. A further question is whether the mapping process as described earlier, would not simply pass the high-health, low-growth solution as it moves away from the starting point $g=0$, i.e. would the high-health, low-growth solution be unstable if there are two points of intersection between $\mathrm{g}=\mathrm{g}(\mathrm{v})$ and $\mathrm{v}=\mathrm{z}(\mathrm{g})$. We have not looked into this matter yet.

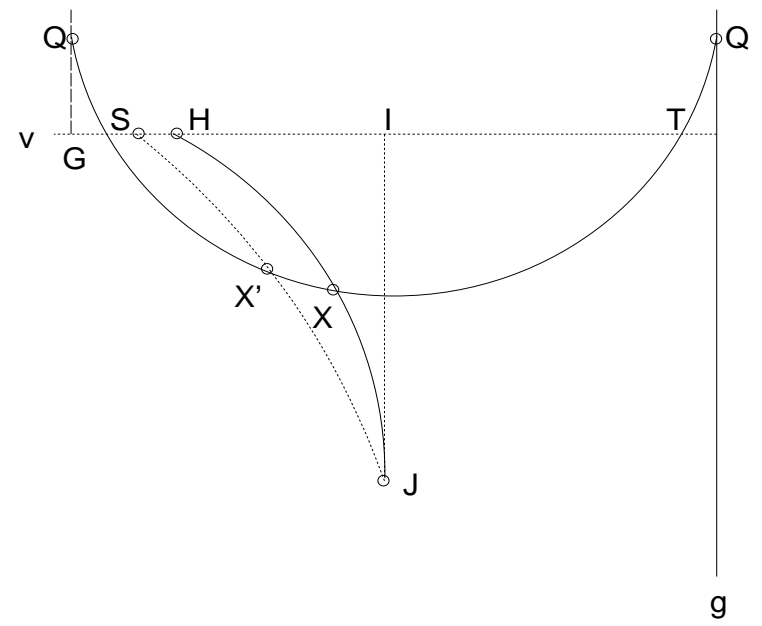

Figure F.4 A rise in $\gamma$

Now consider a change in $\beta$. This raises $v$ for any value of $f$. The effects on $v=z(g)$ are therefore comparable to those of a rise in $\gamma$. But a rise in $\beta$ would also move the extremum of $g(v)$ to the left, without altering the points of intersection of $g(v)$ with the vertical axes at $v=0,1$. Hence the entire curve $v=z(g)$ would become more skewed to the left, whereas the 'flat' part of $g=g(v)$ would shift to the left too. This means that $v$ would be affected to a relatively large extent, while $g$ would hardly be affected at all. In fact, $g$ could even increase, because a rise in $\beta$, would also raise the value of $g(v)$ in $v=\beta$, and hence would cause the curve $g(v)$ not only to become more skewed to the left, but also to be stretched in a downward direction. So a rise in $\beta$ may have definite positive effects on both average health-levels as well as on growth. 


\section{Annex G Model Listing}

The variable names used in the model are the same as in the text. The other names are self explanatory. Note that $P_{-}(A, B)$ is equal to $A$ to the power of $B$, for positive $A$, while IF_ELSE_ $(A, B, C)$ is equal to $B$ when $A<>0$ and to $C$ when $A=0$. AND_, OR_, EQ_are Boolean functions which take the value 1 if true, and 0 if false. So, AND_ $(A, B)=1$ if $A<>0$ and $B<>0$, while OR_ $(A, B)=1$ if $A<>0$ or $B<>0$. $E Q \_(A, B)=1$ if $A=B$, otherwise $E Q \_(A, B)=0$. LE_ $(A, B)=1$ if $A<=B, 0$ otherwise. Iter is a variable which is equal to the number of Gauss-Seidel iterations performed until the moment of evaluation of the calling statement. Iter $=0$ is the very first iteration. Note that the equation for $g=g(v)$ lets the mapping process as its is implicitly defined by the Gauss-Seidel procedure start at highest value of $\mathrm{g}$ which is possible. Hence, given the convergence properties of the mapping process, we will find the economically relevant solution if there is one.

The labelling of the experiments referred to in the model listing coincides with the labelling used in section 5. The base run is experiment 0 and has been labelled X0_. Experiment 1 has been labelled X1_. Experiment 2 has been run for four different values of the rate of transfer $\pi, 0.0051,0.0052,0.01$ and 0.02 . These runs have been labelled $X 2_{-}$ up to X5_.

\{The Model\}

z1 = beta/(1-beta),

1_h $=1$,

$1 \_a=$ if_else_(and_(eq_(experiment,1.0), ge_(time,200)

,

1-max_dl*(time-200)/100,

$1) * \mathrm{~L}$,

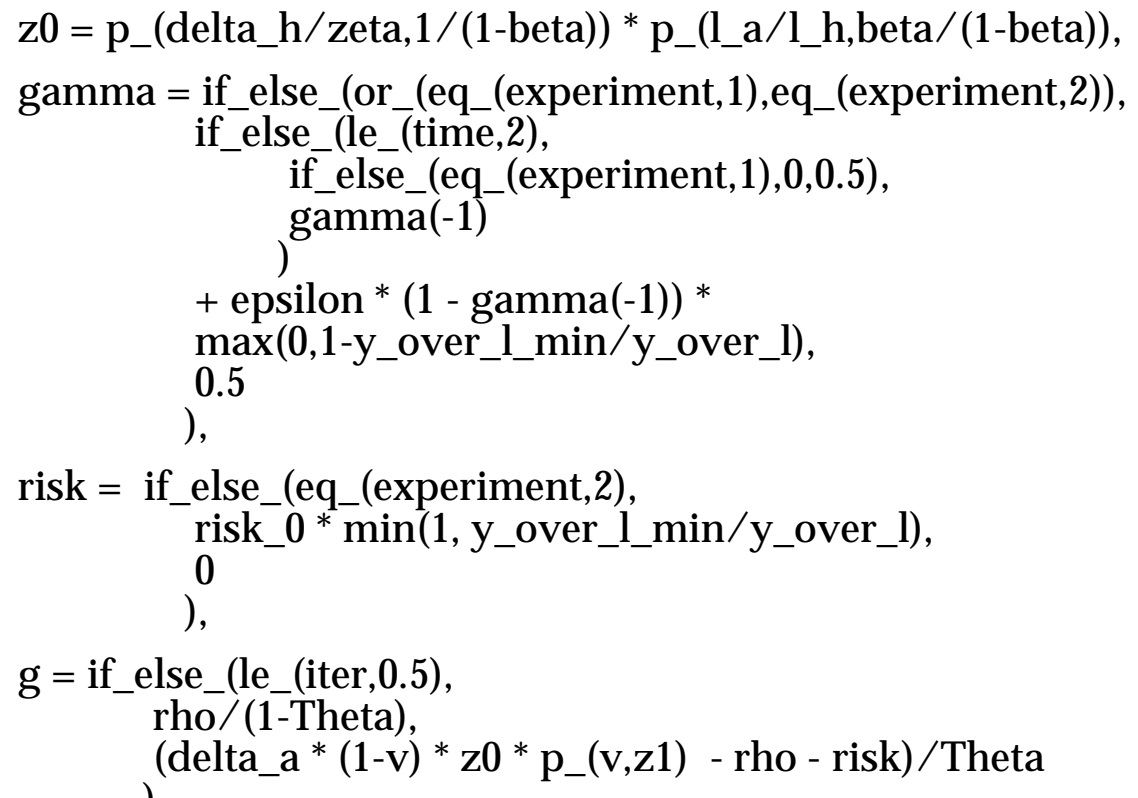




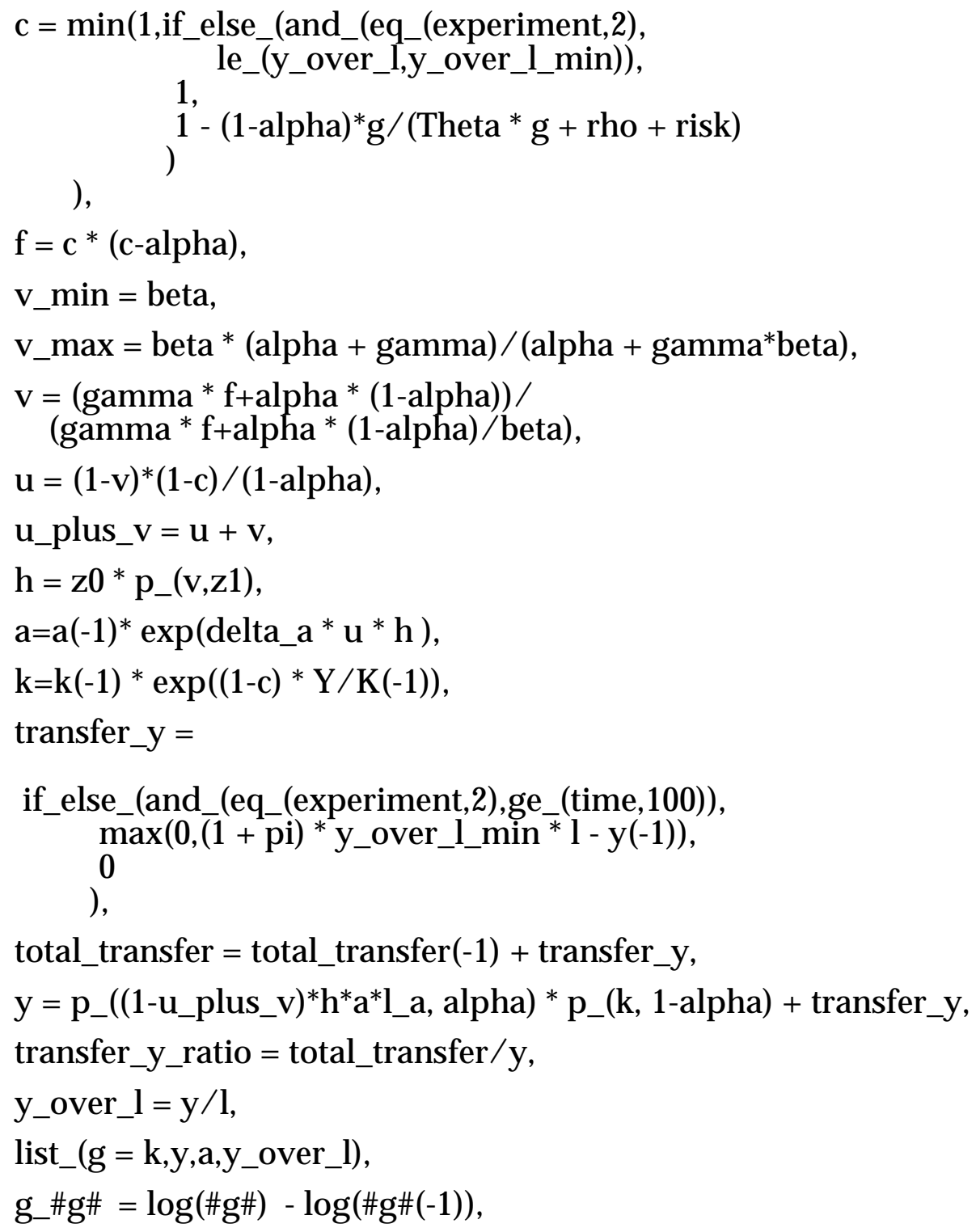




\section{References}

Barro, R.J., and X. Sala-i-Martin, 1995, Economic Growth, MacGraw-Hill.

Baumol, W.J., 1967, 'Macroeconomics of Unbalanced Growth: the Anatomy of Urban Crisis', American Economc Review, Vol. 57, nr. 3, pp. 415-426.

Ehrlich, I. and H. Chuma, 1990, 'A Model of the Deamnd for Longevity and the Value of Life Extension', Lournal of Political Economy, 98, pp. 761-782.

Forster, B.A., 1989, Optimal Health Investment Strategies, Bulletin of Economic Research, 41, pp. 45-57.

Fuchs, V.R., 1982, 'Time Preference and Health: An Exploratory Study', in Fuchs, V.R., ed., Economic Aspects of Health, Conference Report NBER, University of Chicago Press.

Grossman, M., 1972, 'The Demand for Health: a Theoretical and Empirical Investigation', NBER, Occasional Paper 119, Columbia University Press.

Johansson, P.O., and K.G. Lofgren, 1995, 'Wealth from Optimal Health', Journal of Health Economics, 14, pp. 65-79.

Lapre, R.M. and F.F.H. Rutten, 1993, Economie van de Gezondheidszorg, Leerboek voor universitair en hoger beroepsonderwijs, tweede herziene druk, Uitgeverij Lemma BV, Utrecht.

Lucas, R.E, 1988, 'On the Mechanics of Economic Development', Journal of Monetary Economics, 22, pp. 3-42.

Maxwell, R.J., 1981, Health and Wealth, Lexington Books.

Muurinen, J.M., 1982, 'Demand for Health: A Generalised Grossman Model', Journal of Health Economics, 1, pp. 5-28.

Newhouse, J.P., 1977, 'Medical Care Expenditue: a cross-national survey', Journal of Health Economics, 12, pp. 115-125.

Romer, P.M., 1990, 'Endogenous Technological Change', Journal of Political Economy, 5, pp. 71-102.

Solow, R.M., 1956, 'A Contribution to the Theory of Economic Growth', Quarterly Journal of Economics, 70, pp. 65-94. 


\section{Table of Contents}

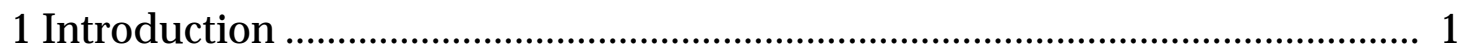

2 Stylised Models of Endogenous Growth and Health Generation ................... 3

3 On the Contribution of Health to Growth and Welfare .................................. 6

4 Parameter Changes and Health and Growth Responses ................................. 11

5 Policy Relevance : Some Illustrative Simulations ............................................. 14

6 Summary and Conclusion ............................................................................. 22

Annex A The Lucas Model ............................................................................. 24

Annex B Health as a Complement to Growth ..................................................... 26

Annex C Health as a Substitute of Growth ........................................................ 28

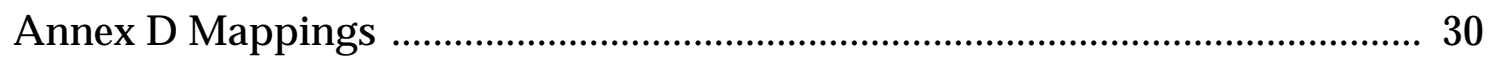

Annex E Parameter Restrictions …………………......................................... 32

Annex F Parameter Changes and System Responses ....................................... 34

Annex G Model Listing ................................................................................... 37

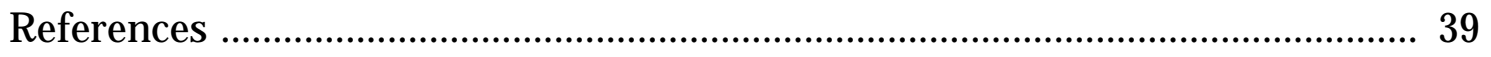

